\title{
Magma Flow through Elastic-Walled Dikes
}

\author{
O. Bokhove ${ }^{1}$, A.W. Woods ${ }^{2}$, A. de Boer ${ }^{1}$ \\ 1 Department of Applied Mathematics, University of Twente, The Netherlands \\ 2 BP Institute for Multiphase Flow, University of Cambridge, U.K.
}

\begin{abstract}
A convection-diffusion model for the averaged flow of a viscous, incompressible magma through an elastic medium is considered. The magma flows through a dike from a magma reservoir to the Earth's surface; only changes in dike width and velocity over large vertical length scales relative to the characteristic dike width are considered. The model emerges when nonlinear inertia terms in the momentum equation are neglected in a viscous, low-speed approximation of a magma flow model coupled to the elastic response of the rock.

Stationary- and traveling-wave solutions are presented in which a Dirichlet condition is used at the magma chamber; and either a (i) free boundary condition, (ii) Dirichlet condition, or (iii) choked-flow condition at the moving free or fixed-top boundary. A choked-flow boundary condition, generally used in the coupled elastic wave and magma flow model, is also used in the convection-diffusion model. The validity of this chokedflow condition is illustrated by comparing stationary flow solutions of the convectiondiffusion and coupled elastic wave and magma flow model for parameter values estimated for the Tolbachik volcano region in Kamchatka, Russia. These free- and fixed-boundary solutions are subsequently explored in a conservative, local discontinuous Galerkin finiteelement discretization. This method is advantageous for the accurate implementation of the choked flow and free boundary conditions. It uses a mixed Eulerian-Lagrangian finite element with special infinite curvature basis function near the free boundary and ensures positivity of the mean aperture subject to a time-step restriction. We illustrate the model further by simulating magma flow through host rock of variable density, and magma flow that is quasi-periodic due to the growth and collapse of a lava dome.
\end{abstract}

Key words: elastic rock walls, magma dynamics, convection diffusion model, discontinuous finite elements

PACS:

\section{Introduction}

We consider the dynamics of viscous, incompressible magma through a dike surrounded by host rock. The dike runs from a magma reservoir deep in the rock to the Earth's surface. At leading order, the rock is modeled as an elastic medium in which the dike width is approximated to be proportional to

Correspondence to: O. Bokhove, Department of Applied Mathematics, University of Twente, 7500 AE, P.O. Box 217, Enschede, The Netherlands. (e-mail: o.bokhove@math.utwente.nl) 
the difference in fluid pressure and the lithostatic pressure caused by the overlying weight of the rock. In this relation, the anisotropy of the stresses is explicitly included by assuming the least principal stress to be horizontal. When changes of the flow over large vertical scales relative to the width or cross section of the aperture are considered, the flow across the aperture is locally in approximate viscous equilibrium. For simplicity, we assume the viscosity to be constant. Hence, the mass and momentum equations averaged over this cross section yield a convection-diffusion equation after inertia terms in the momentum equation have been neglected in favor of this so-called lubrication approximation. The resulting model equals the fracture-propagation model of [5] for the limit of isotropic stresses in the rock once the influence of an edifice load at the surface is neglected. In contrast, our simplified model is still (asymptotically) valid for small edifice loads and is more amenable to mathematical analysis.

In particular, stationary and traveling-wave solutions are derived for the convection-diffusion model, either analytically or by numerically solving the relevant ordinary-differential equations. Solutions are derived for both a free and fixed top boundary; the first describing magma driving its way along a pre-existing crack to the surface, and the latter describing the magma flow for (time-dependent) pressure conditions at the surface. In both cases, the (time-dependent) pressure condition at the magma chamber is prescribed. For high velocities, inertial terms become important, and a momentum and a continuity equation describe the leading-order flow in an elastic dike. The most appropriate boundary condition at the top appears to be the condition of choked or critical flow at the surface. The speed of the flow then matches the velocity of the elastic waves. Despite the lack of inertial terms, the condition of criticality at the top also seems appropriate for the convection-diffusion equation: it could be viewed as the outcome of an analysis in a boundary layer near the surface of the inertial, elastic-flow equations. Steady-flow solutions aiming to represent an eruption of the Tolbachik volcano region in Kamchatka, Russia, show that the solutions of both models are nearly equal when the flow is enforced to be critical at the top ( $c f$., the inertial, elastic-flow solutions in [11]).

These high-resolution exact and lower-dimensional numerical solutions are subsequently used to verify a novel application and extension of a conservative, local discontinuous Galerkin finite-element method for convection-diffusion equations (building on [3]). First, the extended numerical approach uses an upwind flux to ensure positivity of the mean aperture subject to a time-step restriction and is additionally designed to satisfy $L_{2}$-stability for suitable boundary conditions. Second, the asymptotic solution at the free boundary or front is used as a basis function in the time-dependent free-boundary element because the solution at this free boundary is singular and can, therefore, not be matched easily and satisfactorily in any numerical method and by the usual polynomial basis and test functions in discontinuous Galerkin methods in particular. The free-boundary treatment uses fixed Eulerian elements away from the free boundary and a mixed Eulerian-Lagrangian element at the free boundary akin to the flooding and drying approach in [1]. Although in one dimension an entirely Lagrangian treatment is feasible, the presented mixed Eulerian-Lagrangian elements are better suited for extension to two-dimensional flow through a magma dike averaged only over its narrow width. The consideration of such two-dimensional flows through a dike instead of a (nearly) symmetric flow through a horizontally uniform dike is important for applications in which the influence of local sources and sinks of magma in a dike needs to be investigated. Such evolving asymmetric flows in a dike can occur because of heterogeneities in the surrounding host rock (near the free surface), horizontal pressure distributions in the magma chamber, or existing subsurface drifts intersected by a rising magmatic dike.

Verification of the numerical model is performed by a comparison with the high-resolution, stationary and traveling-wave solutions for both the free- and fixed-boundary cases. Finally, the model is explored further by simulating the effects of varying rock density and a suddenly imposed horizontal array of sinks at $300 \mathrm{~m}$ depth on the magma flow, and the growth of a lava dome caused by magma flow through a dike and its collapse when a critical height has been reached.

The organization of this paper is as follows: the convection-diffusion model is introduced in section 2 and the numerical approach is developed in section 3 and Appendix A. Stationary and travelingwave solutions are derived in section 4.1, and numerical explorations are provided in sections 4.2-4.4. Finally, the conclusion is in section 5 . 


\section{Elastic Wall-Fluid Equations}

We investigate the flow of an incompressible fluid with a density, $\rho_{m}$, through a vertical aperture of variable width, $b$. The aperture, $b$, is narrow enough, relative to the vertical scales being considered, that we consider only the averaged velocity, $u$, along the vertical. In that case, the flow profile at each cross section is assumed to be close to its viscous equilibrium even though we allow smooth and sufficiently slow variations in the vertical and in time. The averaged mass and momentum equations are

$$
\begin{gathered}
\partial_{t}\left(\rho_{m} b\right)+\partial_{z}\left(\rho_{m} b u\right)=0 \\
\rho_{m} \partial_{t} u+\rho_{m} u \partial_{z} u=-\partial_{z} p-\rho_{m} g-\gamma \mu u / b^{2}
\end{gathered}
$$

with time $t$, vertical coordinate $z$, magma pressure $p$, gravitational acceleration $g$, and viscosity $\mu$. The last, frictional term in (2.1b) matches the friction of an incompressible flow assumed to be in local equilibrium. These frictional coefficients follow from considering steady, viscous flow in a planar configuration, for example for a flow in a two-dimensional channel $\gamma=12$. The system is closed by relating the aperture, $b$, to the pressure in the fluid and the pressure in the solid at the wall.

In the geophysical application considered, the medium around the aperture is the host rock. The aperture, $b$, is then proportional to the difference in the fluid, $p$, and wall pressure, $p_{r}$. A sketch of the configuration is provided in Fig. 2.1. Assuming an elastic response of the rock surrounding the aperture $(c f .,[5])$, we take

$$
b=\left\{\begin{array}{cl}
\lambda\left(p-\kappa p_{r}\right) & \text { if } p \geq \kappa p_{r} \\
0 & \text { if } p<\kappa p_{r}
\end{array}\right.
$$

with $\lambda$ describing the elasticity of the host medium. This elastic response is valid when the aspect ratio between horizontal and vertical length scales, and the aspect ratio, $b / a$, of the semi-axes of a thin elliptical fracture are (asymptotically) small. Locally, we thus assume the dike cross section to be elliptic, and locally ignore the variation in the vertical. Hence, we can use, asymptotically, the exact solution of elliptical cross section under uniform pressure in a linear elastic medium which is uniform in the vertical direction. The parameter, $0<\kappa \leq 1$, is introduced because the pressure in the rock does not have to be isotropic, which means that the horizontal stresses, causing the opening or closing of the dike, can be smaller than the vertical one, and $\kappa$ will denote the ratio between the horizontal and the vertical stresses. In principle, the anisotropy parameter $\kappa$ should be deduced form observations, which indeed indicate that anisotropies in horizontal and vertical stresses can be existent [7]. Thus, $\kappa$ could be a function of the vertical coordinate $z$ as well. A transition from brittle to ductile behavior of the host rock often occurs at a depth of approximately $10 \mathrm{~km}$. At depths larger than $10 \mathrm{~km}$ we expect the rock to be close to isotropic, so $\kappa \approx 1$, while at depths lower than $10 \mathrm{~km}$ the anisotropy can be quite large, so values of $\kappa=0.3-0.5$ are even possible, see [7]. For simplicity, we keep $\kappa$ fixed in the results presented. We assume the wall pressure $p_{r}$ to be in lithostatic balance

$$
\partial_{z} p_{r}=-\rho_{r} g
$$

with $\rho_{r}=\rho_{r}(z)$ the density of the rock.

We note that the elastic response (2.2) is a simplified model of the Earth's crust, designed to illustrate some of the principles. In practice, the elastic strength may vary with depth owing to the brittle-ductile trasnsition in the crust, and the near surface conditions will become two dimensional, once the length scale of the horizontal along-dike variations become comparable to the distance to the surface (see [11]). However, in this paper, in which we explore aspects of the elasticity on the magma flow, we work with the idealized one-dimensional model. Later in the paper, we introduce calculations which relate to Tolbachik volcano in Kamchatka, and again, these should be viewed as a simplified picture of the full situation.

Hereafter, we will only consider the incompressible case with $\rho_{m}$ constant, see [11] for the (steady state) compressible case. Hence, from (2.1)-(2.3), we obtain

$$
\begin{gathered}
\partial_{t} b+\partial_{z}(b u)=0 \\
\partial_{t} u+u \partial_{z} u=-\frac{1}{\rho_{m} \lambda} \partial_{z} b+\left(\kappa \rho_{r}-\rho_{m}\right) g / \rho_{m}-\gamma \mu u /\left(\rho_{m} b^{2}\right) .
\end{gathered}
$$




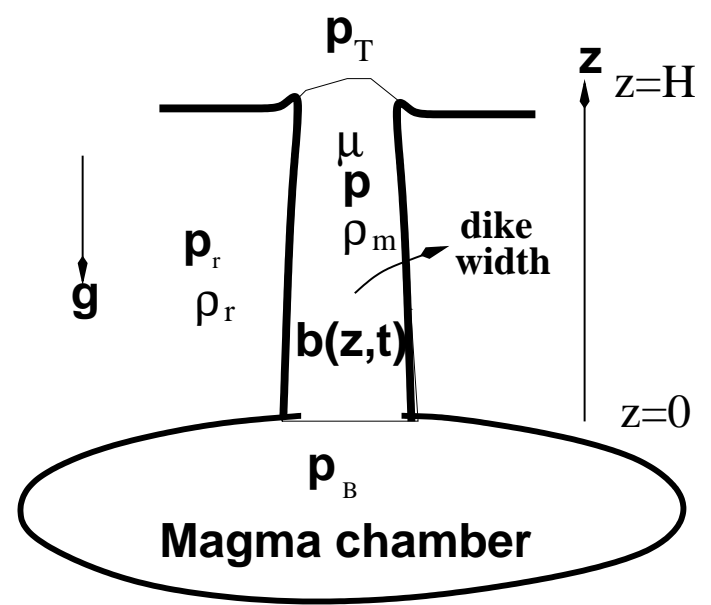

Fig. 2.1. Configuration sketch of the geophysical application with a magma chamber, dike of aperture, $b(z, t)$, and lava dome. The ambient pressure, $p_{T}$, is atmospheric.

It is a system that supports elastic waves with speed $c_{e}=\sqrt{b /\left(\rho_{m} \lambda\right)}($ see, e.g., [9]). The flow of magma in the dike is generally subcritical with $0<u \leq c_{e}$, except at the Earth's surface where it is modeled to be critical (i.e., with $u=c_{e}$ ).

\subsection{Elastic Convection-Diffusion Equations}

Since the flow is viscously dominated with a small Reynolds number of about $2.5-25$, we can ignore the inertia term on the left-hand side of the momentum equation $(2.4 \mathrm{~b})$ to obtain

$$
u=-\frac{b^{2}}{\gamma \mu \lambda} \partial_{z} b+\frac{g b^{2}}{\gamma \mu}\left(\kappa \rho_{r}-\rho_{m}\right)
$$

with $\rho_{m}$ constant but with $\rho_{r}=\rho_{r}(z)$ the variable density of the host rock. Note that this lubrication approximation is used locally under the assumption of slow variations in the vertical, just like we used the exact solution for a crack of elliptical cross section extending infinitely in the vertical direction. On the longer vertical scale, we do allow for variations in the velocity and width. After combining (2.5) with the mass equation, (2.4a), we arrive at the following convection-diffusion equation

$$
\partial_{t} b+\partial_{z}\left(\alpha b^{3}-\beta b^{3} \partial_{z} b\right)=0
$$

with the dimensional expressions for $\alpha=\alpha(z)$ and nonnegative $\beta$ given by

$$
\alpha(z)=g\left(\kappa \rho_{r}(z)-\rho_{m}\right) /(\gamma \mu) \quad \text { and } \quad \beta=1 /(\gamma \mu \lambda) \geq 0 .
$$

For positive buoyancy, $\alpha>0$, and for an overpressure in the magma chamber, the magma is driven upward against the frictional losses, while for negative buoyancy, $\alpha<0$, only the overpressure in the magma chamber acts to overcome the negative buoyancy and viscous losses. This convection-diffusion equation is essentially equivalent to the fracture propagation model of [5] (their equation (4.11)) when the edifice load caused by the weight of a lava dome above a pre-existing crack in their model is considered sufficiently (or asymptotically) small and, hence, is ignored. The elasticity of the medium is then $\lambda=a(1-\nu) / G$ for a dike or fracture of elliptical cross section with $G \approx 1.125 \times 10^{9} \mathrm{~Pa}$ as the rigidity, $\nu \approx 0.25$ as Poisson's ratio, and $a \approx 100 \mathrm{~m}$ as the long semi-axis of the elliptical crack (cf., [5]). To ignore the edifice load, a conservative estimate of the appropriate asymptotic number is the aspect ratio of the height of the lava dome versus the depth of the dike. For a lava dome height of $h_{v}=100-1000 \mathrm{~m}$ and a total conduit depth of $H=3-10 \mathrm{~km}$, this ratio lies between 0.01 and 0.333 . 


\begin{tabular}{c|c|c} 
Variable & Value & Unit \\
\hline$g$ & 9.81 & $\mathrm{~m} / \mathrm{s}^{2}$ \\
$\kappa$ & 0.95 & - \\
$\rho_{r}$ & $2,500-2,900$ & $\mathrm{~kg} / \mathrm{m}^{3}$ \\
$\rho_{m}$ & 2,500 & $\mathrm{~kg} / \mathrm{m}^{3}$ \\
$\lambda$ & $10^{-8}-10^{-6}$ & $\mathrm{~m}^{2} \mathrm{~s}^{2} / \mathrm{kg}$ \\
$D_{0}$ (dike) & $0.5-2$ & $\mathrm{~m}$ \\
$D_{0}$ (conduit) & $5-10$ & $\mathrm{~m}$ \\
$H_{0}$ & $1-10$ & $\mathrm{~km}$ \\
$\alpha$ (dike) & $(-25,50)$ & - \\
$\mu$ & $100-1000$ & $P a s$
\end{tabular}

Table 2.1. Typical values of the variables introduced.

Pinel and Jaupart ([5]) considered the case in which the ratio of the semi-axes of the ellipse is $1: 100$, which results in the symmetric dike model used presently.

After scaling the convection-diffusion equation (2.6) using $b=D_{0} b^{\prime}, t=T_{0} t^{\prime}, z=H_{0} z^{\prime}, H=H_{0} H^{\prime}$, and $T_{0}=\gamma \mu \lambda H_{0}^{2} / D_{0}^{3}$, and dropping the primes, we find (2.6) with (dimensionless) $\beta=1$ and $\alpha(z)=g\left[\kappa \rho_{r}(z)-\rho_{m}\right] \lambda H_{0} / D_{0}$. Typical values of the variables are provided in Table 2.1 which results in an approximate range $\alpha \in(-25,25)$ with typically $\alpha \in(0.1,1)$.

We assume that $u \geq 0$ so the boundary at $z=0$ is an inflow boundary. The dimensionless flow domain $\Omega_{f}=\{z \in(0, H) \mid b(z, t)>0\}$ is a subset of the total domain $\Omega=\{z \in(0, H)\}$, and is smaller when the dike closes $(b=0)$ before it reaches the top. We consider the following combinations of boundary conditions: (i) The pressures at the magma chamber and the exit, and hence the bottom and top width, are specified yielding Dirichlet boundary conditions, $b(0, t)=b_{B}(t)$ and $b(H, t)=b_{T}(t)$. (ii) The pressure at the magma chamber is specified, $b(0, t)=b_{B}(t)$, and the flow at the exit is critical

$$
u(H, t)=\sqrt{b_{T} /\left(\rho_{m} \lambda\right)} \quad \text { (dimensional) or } \quad u(H, t)=\gamma_{c} \sqrt{b} \quad \text { (dimensionless) }
$$

with $\gamma_{c}=\sqrt{\lambda / \rho_{m}} \gamma \mu H_{0} / D_{0}^{5 / 2}$ (using $U_{0}=H_{0} / T_{0}$ ). The width will be smallest and the flow speeds largest at the exit, which implies that the inertial terms may not be neglected near the exit. For the elastic flow model (2.4) generally the choked-flow condition is used (see [10]), because at the exit flow conditions are expected to be critical. By using this choked-flow condition arising from the system (2.4) at the exit also for the convection-diffusion equation, we effectively match this equation to the system (2.4) in an asymptotically thin layer. The approach is supported by a comparison of steady state solutions of both systems (the convection-diffusion equation and the elastic flow model) based on parameter values for the Tolbachik volcano region in Kamchatka, Russia, shown in the results (Fig. 4.5). (iii) The pressure at the magma chamber is specified, $b(0, t)=b_{B}(t)$, and there is a free boundary at $z=z_{r}(t)<H$ where $b\left(z_{r}(t), t\right)=0$. Since $u=\alpha b^{2}-\beta b^{2} \partial_{z} b$, we find at such a free boundary that

$$
\mathrm{d} z_{r}(t) / \mathrm{d} t=u_{r}(t)=u\left(z_{r}(t), t\right)=\lim _{b \downarrow 0}\left(\alpha b^{2}-\beta b^{2} \partial_{z} b\right)=-\beta \lim _{b \downarrow 0}\left(b^{2} \partial_{z} b\right)
$$

The free boundary in case (iii) will evolve into case (i) or (ii) when it reaches the exit. This exit position at $z=H(t)$ may be time dependent when a lava dome is growing or collapsing above the original exit position $H(0)$. As initial condition, we have $b(z, 0)=b_{i}(z)$.

Next, we investigate in detail what happens at such a free boundary. A front at $z=z_{r}(t)$ may exist where the aperture, $b=0$, with $b>0$ for an interval, $0<z<z_{r}(t)$. When $b \downarrow 0$ as $z \uparrow z_{r}(t)$, the velocity limits to

$$
\lim _{b \downarrow 0} u=\lim _{b \downarrow 0}\left(\alpha b^{2}-\beta b^{2} \partial_{z} b\right)=u_{r}(t) .
$$

Note that the advective term $\alpha b^{2}$ at the front does not contribute to the frontal velocity since $b=0$ at the front $z_{r}(t)$, while the term $b^{3} \partial_{z} b$ is nonzero at the front even though $b=0$ there. Because the velocity, $u$, must be finite, the slope of the aperture at the front has to be infinite or horizontal. The solution Ansatz at the front is that $b=D_{0}\left(z_{r}(t)-z\right)^{1 / 3}$ with $D_{0}=D_{0}(t)$ constant in space, giving a 
finite frontal velocity, $u_{r}(t)=\beta D_{0}^{3}$. Alternatively, consider a boundary layer with scaled coordinate, $\eta=\left(z_{r}-z\right) / \epsilon$, and time, $\tau=t / \epsilon$, with $\epsilon \ll 1$. Applying this change of coordinates in (2.6), expanding $b=b^{(0)}+\epsilon b^{(1)}+O\left(\epsilon^{2}\right)$, and evaluating at leading order $1 / \epsilon^{2}$ yields

$$
\partial_{\eta} b^{(0)} \frac{\mathrm{d} z_{r}}{\mathrm{~d} \tau}=\beta \partial_{\eta}\left(b^{(0)^{3}} \partial_{\eta} b^{(0)}\right) .
$$

Integration over $\eta$ gives

$$
b^{(0)} \frac{d z_{r}}{d \tau}-Q_{f}=\beta b^{(0)^{3}} \partial_{\eta} b^{(0)} .
$$

If $Q_{f} \neq 0$, the discharge, $u b$, is finite at the front. The velocity, $u$, is then infinite at and large near the front, which is unphysical and mathematically inconsistent. In the derivation of the convectiondiffusion model, the inertia terms were considered negligible in the momentum equation, (2.1b), but for large velocities these terms should be included. Hence, we have to consider $Q_{f}=0$ to be consistent. Further integration of (2.12) yields the free-boundary condition and the asymptotic aperture profile at the front:

$$
\mathrm{d} z_{r} / \mathrm{dt}=u_{r}(t)=\frac{1}{3} \beta D_{0}^{3} \quad \text { and } \quad b=D_{0}(t)\left(z_{r}(t)-z\right)^{1 / 3}
$$

with integration constant $D_{0}=D_{0}(t)$. This asymptotic profile will be used as a time-dependent basis function in a free-boundary element of the discontinuous Galerkin finite-element method. Finally, we note that the total amount of mass in the flow domain depends on the inflow and outflow at the boundaries.

\section{Finite-Element Discretization}

The following finite-element discretization is described in sufficient detail to accommodate the readers less familiar with discontinuous Galerkin methods. Technical parts on stability and the specific choice of the numerical flux are found in the appendix. The reasons to use this more complex method above a finite difference method are as follows. (i) It allows more accurate tracking of moving fronts in a mixed Eulerian and Lagrangian scheme with infinite curvature (2.13) at the front. (ii) The choked-flow condition is implemented easily. (iii) It is advantageous in more complex (moving) flow geometries in two dimensions in which also the horizontal variation along the dike is permitted.

We reformulate (2.6) as follows

$$
\partial_{t} b+\partial_{z} F_{b}=0 \quad \text { and } \quad \beta q+\partial_{z} F_{q}=0
$$

with fluxes

$$
\text { F1: } \quad F_{b}=u b=\left(\alpha b^{2}+\beta \sqrt{b} q\right) b \quad \text { and } \quad F_{q}=\frac{2}{5} \beta b^{5 / 2}
$$

or

$$
\mathrm{F} 2: \quad F_{b}=u_{s} b^{3 / 2} \equiv\left(\alpha b^{3 / 2}+\beta q\right) b^{3 / 2} \quad \text { and } \quad F_{q}=\frac{2}{5} \beta b^{5 / 2} .
$$

This formulation is concisely written as

$$
\partial_{t} \tilde{w}+w^{\dagger}+\partial_{z} F=0
$$

with variables $\tilde{w}=(b, 0)$ and $w^{\dagger}=(0, \beta q)$, and fluxes $F=\left(F_{b}, F_{q}\right)$.

\subsection{Finite elements}

We define a tessellation, $\mathcal{T}_{h}$, of $N_{\mathrm{e}}$ elements, each denoted by $K_{k}$, in the spatial flow domain, $\Omega_{f} \subseteq \Omega$, with boundary $\partial \Omega_{f}$ :

$$
\mathcal{T}_{h}=\left\{K_{k}: \bigcup_{k=1}^{N_{\mathrm{e}}} \bar{K}_{k}=\bar{\Omega} \text { and } K_{k} \cap K_{k^{\prime}}=\emptyset \text { if } k \neq k^{\prime}, 1 \leq k, k^{\prime} \leq N_{\mathrm{e}}\right\} ;
$$


here $K_{k}$ may be a time-dependent free-boundary edge element when it includes the front where the aperture, $b=0$, and $\bar{K}_{k}$ denotes the closure of $K_{k}$, and likewise for $\bar{\Omega}$. Element $K_{k}$ runs from node $z_{k}$ to node $z_{k+1}$, and has a length of $\left|K_{k}\right|=z_{k+1}-z_{k}$. We consider finite-element discretizations of (3.4) with approximations, $w_{h}=\left(b_{h}, q_{h}\right)$, to the state vector, $(b, q)$, and basis functions, $v=\left(v_{b}, v_{q}\right)$, to be introduced. The discretization is such that $b_{h}, q_{h}$, and $v_{b, q}$ (when $K_{k}$ is not an edge element) belong to the broken space

$$
V_{h}=\left\{v|v|_{K_{k}} \in P^{d_{P}}\left(K_{k}\right), k=1, \ldots, N_{\mathrm{e}}\right\},
$$

in which $P^{d_{P}}\left(K_{k}\right)$ denotes the space of polynomials in $K_{k}$ of degree $d_{P}$. For edge elements, $b_{h}$ consists of an asymptotic solution analogous to (2.13).

\subsection{Weak formulation}

For the moment, we ignore the time dependence of basis and test functions in the edge elements. We multiply (3.1) by test functions, $v=\left(v_{b}(z), v_{q}(z)\right)$, integrate by parts for each individual and isolated element, and then add the contribution from all elements to obtain the following weak formulation

$$
\begin{aligned}
& \sum_{k=1}^{N_{e}}\left\{\int_{K_{k}} v_{b} \frac{\mathrm{d} b_{h}}{\mathrm{~d} t} \mathrm{~d} z+\left[F_{b}\left(z_{k+1}^{-}\right) v_{b}\left(z_{k+1}^{-}\right)-F_{b}\left(z_{k}^{+}\right) v_{b}\left(z_{k}^{+}\right)\right]-\int_{K_{k}} F_{b} \partial_{z} v_{b} \mathrm{~d} z\right\}=0 \\
& \sum_{k=1}^{N_{e}}\left\{\int_{K_{k}} \beta v_{q} q_{h} \mathrm{~d} z+\left[F_{q}\left(z_{k+1}^{-}\right) v_{q}\left(z_{k+1}^{-}\right)-F_{q}\left(z_{k}^{+}\right) v_{q}\left(z_{k}^{+}\right)\right]-\int_{K_{k}} F_{q} \partial_{z} v_{q} \mathrm{~d} z\right\}=0,
\end{aligned}
$$

where $v_{b, q}\left(z_{k+1}^{-}\right)=\lim _{z \uparrow z_{k+1}} v_{b, q}(z, t)$ and $v_{b, q}\left(z_{k}^{+}\right)=\lim _{z \downarrow z_{k}} v_{b, q}(z, t)$. (We only denote these dependencies explicitly when confusion may arise.) Hence, the fluxes at the faces arising in elements $K_{k}$ are evaluated inside each element.

Let

$$
[[b]]=b_{+}-b_{-} \quad \text { and } \quad \bar{b}=\left(b_{+}+b_{-}\right) / 2
$$

denote the jump and mean in the quantity $b$ at $z_{k+1}$ with the trace values $b_{-}=\lim _{z \uparrow z_{k+1}} b(z)$ and $b_{+}=\lim _{z \downarrow z_{k+1}} b(z)$. Consider the flux at a point $z_{k+1}$. Because the elements are isolated from each other in $(3.7), b_{-}:=b\left(z_{k+1}^{-}\right) \neq b\left(z_{k+1}^{+}\right)=: b_{+}$, and, consequently, the flux $F\left(z_{k+1}^{-}\right) \neq F\left(z_{k+1}^{+}\right)$in general. The heart of the discontinuous Galerkin numerical method hinges, therefore, on the choice of a numerical flux. To enforce communication and conservation of the fluxes between each element in (3.7), the fluxes $F\left(z_{k}^{+}\right)$and $F\left(z_{k+1}^{-}\right)$at the faces of $K_{k}$ are replaced by numerical fluxes $\tilde{F}\left(z_{k}\right)=\tilde{F}\left(w_{k}^{-}, w_{k}^{+}\right)$and $\tilde{F}\left(z_{k+1}\right)=\tilde{F}\left(w_{k+1}^{-}, w_{k+1}^{+}\right)$. For details, see Appendix A.

\subsection{Geometry and polynomial basis functions}

The bounded interval $\Omega:=[0, H] \subset \mathbb{R}$ is partitioned by $N_{e}+1$ "regular" faces (points in one dimension) $\mathcal{E}:=\left\{z_{k}\right\}_{k=0}^{N_{e}}$ and into $N_{e}$ "regular" elements. It is convenient to introduce a reference element in one dimension, $\hat{K}=[-1,1]$, and define the mapping $F_{K}: \mathbb{R} \rightarrow \mathbb{R}$ between the reference element, $\hat{K}$, and element $K_{k}$ as follows: $z=F_{K_{k}}(\zeta)=\sum_{m=1}^{2} z_{k, m} \chi_{m}(\zeta)=\hat{z}_{k}+\left|K_{k}\right| \zeta / 2$, where $z_{k, 1}=z_{k}$ and $z_{k, 2}=z_{k+1}$ are the left and right end points of element $K_{k}=\left(z_{k}, z_{k+1}\right)$. The shape functions are $\chi_{1}(\zeta)=(1-\zeta) / 2$ and $\chi_{2}(\zeta)=(1+\zeta) / 2$. Note that $\hat{z}_{k}=\left(z_{k, 1}+z_{k, 2}\right) / 2,\left|K_{k}\right|(t)=\left(z_{k, 2}-z_{k, 1}\right)$. On the basis element $\hat{K}$, we define basis functions

$$
\hat{\varphi}_{0}(\zeta)=1 \text { and } \hat{\varphi}_{m}(\zeta)=\zeta^{m} \text { for } m=1, \ldots, d_{P}
$$

Finally we relate the local basis functions in $\hat{K}$ to the basis functions in $K_{k}$ as follows:

$$
\hat{\varphi}_{n}(\zeta)=\hat{\varphi}_{n}\left[F_{K_{k}}^{-1}(z, t)\right]=\varphi_{n, k}(z, t) \quad \text { for } \quad n=0, \ldots, d_{P} .
$$

In principle, elements can also be time dependent by allowing the nodes to move in time. We distinguish fixed interior elements where $b>0$ and edge elements where $b=0$ at one of the nodes. 
The vector $(b, q)$ in both cases $\mathrm{F} 1$ and $\mathrm{F} 2$ and test functions, $v$, are approximated in each (timedependent) element, $K_{k}$, by their polynomial approximations $\left(b_{h}, q_{h}\right)$ and $v_{b, q}$, as follows:

$$
b_{h}(z, t)=\sum_{m=0}^{d_{P}} \hat{B}_{m}\left(K_{k}, t\right) \psi_{m}(z, t), \quad v_{b, q}(z)=\sum_{m=0}^{d_{P}} \hat{v}_{m}\left(K_{k}\right) \psi_{m}(z, t)
$$

with polynomial basis functions $\psi_{m}(z, t) \in P^{d_{P}}\left(K_{k}\right)$. These are chosen such that

$$
\begin{aligned}
\hat{B}_{0} & =\bar{B}\left(K_{k}, t\right)=\int_{K_{k}} b(z, t) \mathrm{d} z /\left|K_{k}\right| \text { and } \\
\psi_{m, k}(z, t) & =\left\{\begin{array}{cr}
1 & \text { if } m=0 \\
\varphi_{m, k}(z, t)-\int_{K_{k}} \varphi_{m, k}(z, t) \mathrm{d} z /\left|K_{k}\right| & \text { if } m \geq 1
\end{array}\right\} .
\end{aligned}
$$

Note that $\psi_{1, k}=\zeta$. Likewise, we approximate $q$ with $q_{h}$.

\subsection{Weak formulation and finite-element discretization}

Taking $d_{P}=1$ in (3.11), we approximate $b$ on $K_{k}$ by a mean and a slope

$$
b_{h}(z, t)=\bar{B}_{k}+\hat{B}_{k} \psi_{1, k}(z, t) \quad \text { and } \quad v_{h}(z)=\bar{W}_{k}+\hat{W}_{k} \psi_{1, k}(z, t)
$$

with $\bar{B}_{k}=\bar{B}\left(K_{k}, t\right)$ the mean, and $\hat{B}_{k}=\hat{B}\left(K_{k}, t\right)$ the slope, and likewise for $q_{h}$. For the moment, we restrict to the case where the nodes remain fixed. Since $\bar{W}_{k}$ and $\hat{W}_{k}$ are arbitrary, we obtain, after substituting (3.12) into (3.7), the following equations for the mean and fluctuating part

$$
\begin{aligned}
& \left|K_{k}\right| \frac{\mathrm{d} \bar{B}_{k}}{\mathrm{~d} t}+\tilde{F}_{b}\left(z_{k+1}\right)-\tilde{F}_{b}\left(z_{k}\right)=0 \\
& \frac{\left|K_{k}\right|}{3} \frac{\mathrm{d} \hat{B}_{k}}{\mathrm{~d} t}+\left[\tilde{F}_{b}\left(z_{k+1}\right)+\tilde{F}_{b}\left(z_{k}\right)\right]-\int_{-1}^{1} F_{b}\left(b_{h}, q_{h}\right) \mathrm{d} \zeta=0 \\
& \beta\left|K_{k}\right| \bar{Q}_{k}+\tilde{F}_{q}\left(z_{k+1}\right)-\tilde{F}_{q}\left(z_{k}\right)=0 \\
& \beta \frac{\left|K_{k}\right|}{3} \hat{Q}_{k}+\left[\tilde{F}_{q}\left(z_{k+1}\right)+\tilde{F}_{q}\left(z_{k}\right)\right]-\int_{-1}^{1} F_{q}\left(b_{h}, q_{h}\right) \mathrm{d} \zeta=0 .
\end{aligned}
$$

The integrals are approximated with a third-order Gauss quadrature rule. Further details of the numerical discretization such as the time discretization, the choice of numerical fluxes, $\tilde{F}_{b}$ and $\tilde{F}_{q}$, and its $L_{2}$-stability are found in the Appendix A.

\subsection{Time discretization: non-negative mean aperture}

The upwind nature of the chosen fluxes is favored to avoid a negative aperture. The idea of a maximum principle (e.g., [4],[1]) can then be used to estimate a suitable time-step restriction such that the mean aperture in each element remains positive.

Define the mean, $\bar{B}_{k}$, and fluctuation, $\hat{B}_{k}$, of $b_{h}$ in element $K_{k}$ as follows

$$
\left.b_{h}\right|_{K_{k}}=\bar{B}_{k}+\zeta \hat{B}_{k}
$$

with $\zeta \in[-1,1]$ the reference coordinate in element $K_{k}$. Using the test function $v_{b}=1$, we obtain the discrete equation for the mean aperture, $\bar{B}_{k}$, in element $K_{k}$

$$
\left|K_{k}\right| \frac{\mathrm{d} \bar{B}_{k}}{\mathrm{~d} t}+\tilde{F}_{b}\left(z_{k+1}\right)-\tilde{F}_{b}\left(z_{k}\right)=0,
$$

where for F1: $\tilde{F}_{b}=\langle u\rangle_{\text {conv }} b_{ \pm}+\langle u\rangle_{\text {diff }} b_{ \pm}$with $\langle u\rangle_{\text {conv }}=\overline{u_{\text {conv }}}=\alpha\left(b_{-}^{2}+b_{+}^{2}\right) / 2$ and

$$
\langle u\rangle_{\mathrm{diff}}=\left\{\begin{array}{ll}
\beta q_{+} \sqrt{b_{-}} \text {if } & q_{+} \geq 0 \\
\beta q_{+} \sqrt{b_{+}} \text {if } & q_{+}<0
\end{array},\right.
$$


and for F2: $\tilde{F}_{b}=\left\langle u_{s}\right\rangle b_{ \pm}^{3 / 2}$ with $\left\langle u_{s}\right\rangle=\overline{u_{s}}$. So if $\overline{u_{\text {conv }}} \geq 0$ we choose $b_{ \pm}\left(z_{k}\right)=b_{-}$and vice versa, and likewise for the diffusive velocity, $\langle u\rangle_{\text {diff }}$, and scaled velocity, $u_{s}$.

The third-order total variation diminishing Runge-Kutta (RK3) method of [6] is used to discretize time (see Appendix A), but we show the analysis for the forward Euler step. Given $b_{k}^{n}$ at time level $t=t_{n}$, then $b_{k}^{n+1}$ at the next time level, $t=t_{n+1}=t_{n}+\Delta t$, is found using intermediate stages $b_{k}^{(1)}$ and $b_{k}^{(2)}$. Consider the flux formulation F2 such that at the face $z_{k+1}: b_{-}=\bar{B}_{k}+\hat{B}_{k}$ and $b_{+}=\bar{B}_{k+1}-\hat{B}_{k+1}$, with a positive velocity, $\left\langle u_{s}\right\rangle^{n}>0$, and a positive aperture, $b^{n}>0$, at time $t_{n}$ for the first forward Euler (an intermediate time stage in the RK3 method). We deduce

$$
\begin{aligned}
0 & <\bar{B}_{k}^{n}\left(1-\frac{2 \Delta t}{\left|K_{k}\right|}\left\langle u_{s}\right\rangle_{k+1}^{n}\right)+\frac{\Delta t}{\left|K_{k}\right|}\left\langle u_{s}\right\rangle_{k}^{n}\left(\bar{B}_{k-1}^{n}+\hat{B}_{k-1}^{n}\right) \leq \bar{B}_{k}^{(1)} \\
& =\bar{B}_{k}^{n}-\frac{\Delta t}{\left|K_{k}\right|}\left\langle u_{s}\right\rangle_{k+1}^{n}\left(\bar{B}_{k}^{n}+\hat{B}_{k}^{n}\right)+\frac{\Delta t}{\left|K_{k}\right|}\left\langle u_{s}\right\rangle_{k}^{n}\left(\bar{B}_{k-1}^{n}+\hat{B}_{k-1}^{n}\right),
\end{aligned}
$$

since $\bar{B}_{k}+\hat{B}_{k} \leq 2 \bar{B}_{k}$ if $\left(b_{h}\right)_{k}>0$. We can ensure positivity of $\bar{B}_{k}^{(1)}$ by limiting the time step in (3.17) such that the coefficients of $\bar{B}_{k}^{n}$ remain positive, as follows

$$
\Delta t_{k} \leq 0.5\left|K_{k}\right| /\left\langle u_{s}\right\rangle_{k+1}^{n}
$$

An overall time step is obtained by taking the smallest time step resulting from the elements $K_{k}$. A similar time step criterion appears at the other intermediate time stages by taking $\langle u\rangle^{(1)},\langle u\rangle^{(2)}$ to eventually ensure that $\bar{B}_{k}^{n+1}>0$. The same analysis can be made for F1. Because we do not know the values at intermediate stages $t^{(1)}$ and $t^{(2)}$ in advance, it may be necessary to restart the time integration at $t_{n}$ with a smaller time step.

In addition, a slope limiter is required such that $\bar{B}_{k} \pm \hat{B}_{k}>0$ at (intermediate) stages. We used the limiter of [2] to limit oscillations around steep gradients. After each completed time step, closure of the aperture so that $\bar{B}_{k} \pm \hat{B}_{k}<0$ for positive mean $\bar{B}_{k}$ would indicate that a closing event has emerged.

\subsection{Free-boundary elements}

In the presented simulations, only one free boundary (point) is considered with $b=0$ for $z>z_{r}(t)$. Consider an underlying fixed mesh of elements and a mesh with flooded elements where $b>0$. The flooded part of the mesh consists of Eulerian finite elements coinciding with the fixed mesh for $z<z_{t e}$ and an Eulerian-Lagrangian moving top element with $k=t e$ and fixed bottom nodes at the left $z=z_{t e}$ and right $z=z_{t e+1}$, and a moving top node with $z_{r}(t)>z_{t e}$.

The aperture in this top element, $k=t e$, is expressed in terms of the asymptotic free boundary solution (2.13) as

$$
b_{h}(z, t)=\frac{4}{3} \bar{B}_{t e}(t) \frac{\left[z_{r}(t)-z\right]^{1 / 3}}{\left|K_{t e}\right|^{1 / 3}},
$$

since $b_{h}$ is approximated by $(2.13)$ and its mean satisfies

$$
\bar{B}_{t e}(t)=\frac{1}{z_{r}-z_{t e}} \int_{z_{t e}}^{z_{r}} b_{h}(z, t) \mathrm{d} z
$$

After substitution in the weak formulation with $v_{b}=1$, but now allowing for time-dependent basis and test functions, mass conservation emerges

$$
\frac{\mathrm{d}\left(\left|K_{t e}\right| \bar{B}_{t e}\right)}{\mathrm{d} t}-\tilde{F}_{b}\left(z_{t e}\right)=0 \quad \text { and } \quad \frac{d z_{r}}{d t}=\frac{64 \beta}{81} \frac{\bar{B}_{k}^{3}}{\left|K_{t e}\right|}
$$

with $\left|K_{t e}\right|=z_{r}(t)-z_{t e}$. Likewise, we can handle a bottom free-boundary element. The discretization of the equation for $q$ remains the same (or can be altered correspondingly), except that the the basis function used for $b$ in a free-boundary element has to be altered.

When the top element becomes too large, for instance, larger than

$$
\left|K_{t e}\right|+0.6\left|K_{t e+1}\right|_{\text {fixed }},
$$


(a)

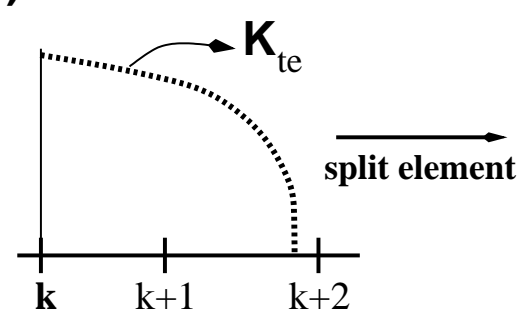

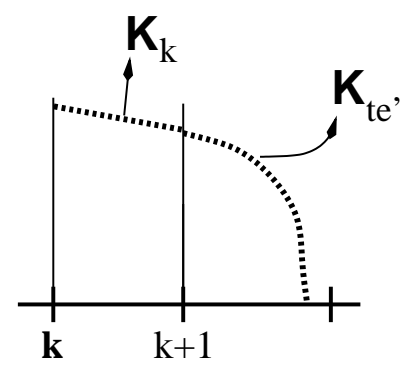

(b)

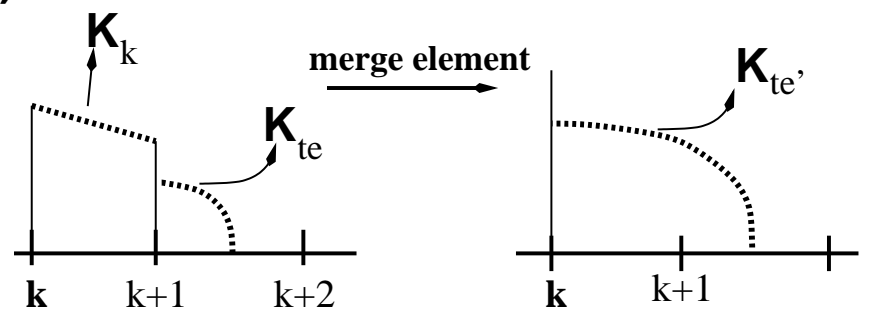

Fig. 3.1. (a) Splitting and (b) merging of the time-dependent top element with expansion, $b=b_{h}(z, t) \propto \bar{B}_{k}(t)\left[z_{r}(t)-\right.$ $z]^{1 / 3}$.

then element $K_{t e}$ becomes a regular fixed element and $K_{t e+1}$ the new time-dependent top element, see Fig. 3.1a. Conservation of mass yields $\bar{B}_{t e}$ and $\bar{B}_{t e+1}$, while $\hat{B}_{t e}$ follows by (numerical) projection. When the top element becomes too small, for instance, less than $0.4\left|K_{t e}\right|_{\text {fixed }}$ of the fixed underlying element, then elements $K_{t e-1}$ and $K_{t e}$ are combined under conservation of mass; see Fig. 3.1b. A new time-dependent top element, $K_{t e-1}$, emerges with $\bar{B}_{t e-1}$, while $\bar{B}_{t e}$ becomes void. In principle, closing events emerging in the middle of the domain can be included as well (in the analogy of the drying events in [1]) but were not required in the presented simulations. Furthermore, the time-step criterion for free-boundary elements is adjusted to include the effect of the moving free-boundary node.

\section{Results}

\subsection{Stationary and Traveling-Wave Solutions}

Substituting the traveling-wave Ansatz, $b=b(\varphi)$, with $\varphi=(z-c t)$ into (2.6) yields

$$
\beta b^{3} b^{\prime}=\alpha b^{3}-c b-Q=f(b)-Q
$$

with $b^{\prime}=\mathrm{d} b / \mathrm{d} \varphi$ and the integration constant $Q$, which equals the flow rate when $c=0$. Several cases will be considered: stationary solutions with $c=0$ and $Q \geq 0$, and traveling-wave solutions with $c \neq 0$. For traveling-wave solutions with a free boundary, we must have a finite velocity at the front, and thus $Q=0$ has to be imposed.

\subsubsection{Stationary solutions}

When $c=0$, we find

$$
\beta b^{3} b^{\prime}=\alpha b^{3}-Q=f(b)-Q .
$$

In Fig. 4.1a, we display $f(b)$ versus $b$ for $\alpha>0$ with root $b=b_{*}$ when $f(b)-Q=0$, while $b>0$ and $Q>0$. We note that $b^{\prime}<0$ when $f(b)<Q$, so if we start with a value $b=b_{B}<b_{*}$ at the bottom $(z=0)$ then the aperture decreases going upward. Otherwise, when $f(b)>Q$, we find $b^{\prime}>0$, 
(a) $\alpha>0, c=0$

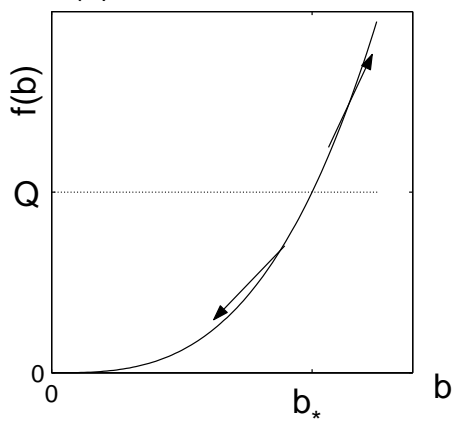

(b) $\alpha<0, c=0$

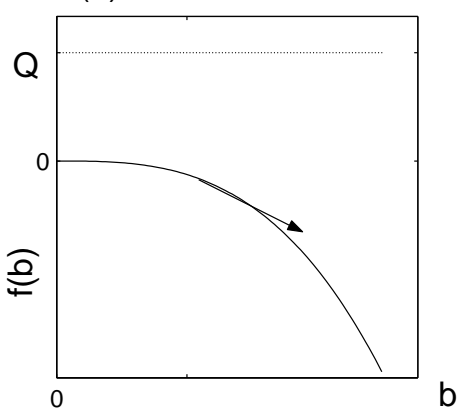

Fig. 4.1. The function $f(b)$ versus $b$ for the stationary cases with $c=0$ and $Q>0$ : (a) for $\alpha>0$, the dike width contracts for $b<b_{*}$ and expands when $b>b_{*}$, and (b) for $\alpha<0$, the dike width only contracts.

and the aperture increases. In Fig. 4.2a, we divide the parameter plane $Q-b_{B}$ with $b_{B}>0$ into an inaccessible region, where the aperture closes before the top at $z=H$, and regions where the aperture expands (right of the dash-dotted line) and contracts (between the two indicated lines). For $\alpha>0$, the buoyancy and the overpressure in the magma chamber act in unison to overcome the viscous losses. These viscous losses are larger for smaller widths and higher flow rates $Q$, for which the gradient of the width decreases more and more as follows from the term $-Q /\left(\beta b^{3}\right)$ in (4.2). Corresponding profiles of $z$ versus $b(z)$ in Fig. 4.2b for various values of $Q$ illustrate some of the permissable profiles. When the aperture becomes asymptotically small at the top, flow speeds become asymptotically large, implying that the neglected inertia terms are no longer negligible. The profile with a critical flow speed, $u=c=\gamma_{c} \sqrt{b}[c f .,(2.8)]$, implies that $Q=u b=\gamma_{c} b_{T}^{3 / 2}$, corresponding to the dashed line in Fig. 4.2b.

In Fig. 4.1b, we display $f(b)$ versus $b$ for $\alpha<0$. When $\alpha<0$, we always find $b^{\prime}<0$ for the relevant cases with $b>0$ and $Q>0$. The parameter plane $Q-b_{B}$ in Fig. 4.3a indicates that only the contracting profiles emerge, as in Fig. 4.3b. For $\alpha<0$, the gradient of the width is always negative as both buoyancy and viscous losses act against the driving overpressure in the magma chamber.

In general, the density of the rock, $\rho_{r}$, may vary mildly with depth (e.g., approximately linearly),

$$
\rho_{r}(z)=(1-z / H) \rho_{0}+\rho_{H} z / H .
$$

We obtain the same equation (4.2), and the qualitative behavior is the same if the sign of $\alpha$ is definite in the domain. For $\alpha(z)>0$, we obtain the parameter space $Q-b_{B}$, as shown in Fig. 4.4 with the corresponding depth-versus-aperture-profiles.

Another representative model of the crustal density arises from the Tolbachik volcano region in Kamchatka, Russia, with

$$
\rho_{r}(z)=\left\{\begin{array}{l}
2,400 \mathrm{~kg} / \mathrm{m}^{3} 26 \mathrm{~km}<z<30 \mathrm{~km} \\
2,600 \mathrm{~kg} / \mathrm{m}^{3} 22 \mathrm{~km}<z<26 \mathrm{~km} \\
2,800 \mathrm{~kg} / \mathrm{m}^{3} 0 \mathrm{~km}<z<22 \mathrm{~km}
\end{array}\right.
$$

So the magma chamber lies at a $30 \mathrm{~km}$ depth. Furthermore, the ratio of minimum to maximum principle stress is taken as $\kappa=0.95$; the viscosity $\mu=100 \mathrm{~Pa} \mathrm{~s}$; the magma density $\rho_{m}=2,600 \mathrm{~kg} / \mathrm{m}^{3}$; and $\lambda=6 \times 10^{-8} \mathrm{~m}^{2} \mathrm{~s}^{2} / \mathrm{kg}$. Steady-state solutions of the inertial, elastic equations (see, [11]) and the convection-diffusion equations are both displayed in Fig. 4.5. They are indistinguishable on the scale shown. Hence, the condition of choked flow in the convection-diffusion model seems appropriate here. The steady-state equation for the elastic flow equations (2.4) with $Q=b u$ is

$$
\left(b / \lambda-\rho_{m} Q^{2} / b^{2}\right) \partial_{z} b=\partial_{z}\left(\frac{1}{2} b^{2} / \lambda+\rho_{m} Q^{2} / b\right)=g\left(\kappa \rho_{r}-\rho_{m}\right) b-\gamma \mu Q / b^{2}
$$

\subsubsection{Traveling-wave solutions}

When $c \neq 0$, traveling-wave solutions emerge. We consider first the most relevant cases with constant $\alpha>0$. In Fig. 4.6, we display $f(b)$ versus $b$ and denoted the roots of $f(b)-Q=0$ by $b_{0}, b_{1}$, or $b_{*}$. 

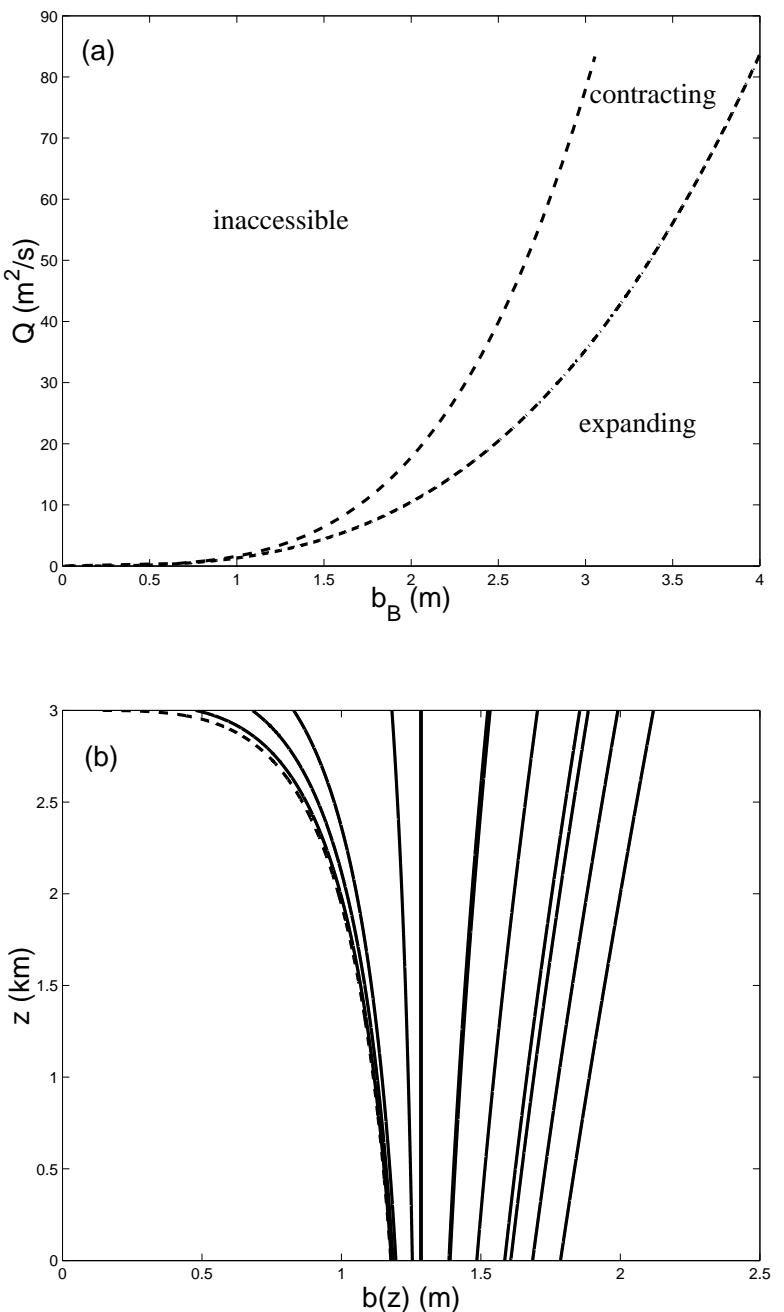

Fig. 4.2. Stationary case $\alpha>0$. (a) The parameter plane $Q-b_{B}$ with $b_{B}>0$ is from left to right divided into an inaccessible, contracting and expanding region. (b) Profiles of the vertical coordinate, $z$, versus the aperture, $b(z)$, of the latter two regions are displayed. The profile with the dashed line indicates the profile with critical speed at the top for which $u=\gamma_{c} \sqrt{b}$ and here $b=b_{T}=0.1245 \mathrm{~m}$ and $Q=2.7778 \mathrm{~m}^{2} / \mathrm{s}$. Parameters are $\alpha=0.4709, H_{0}=3,000 \mathrm{~m}, D_{0}=$ $1 \mathrm{~m}, \rho_{m}=2,500 \mathrm{~kg} / \mathrm{m}^{3}, \rho_{r}=2,800 \mathrm{~kg} / \mathrm{m}^{3}, \kappa=0.95, \lambda=10^{-7} \mathrm{~m}^{2} \mathrm{~s}^{2} / \mathrm{kg}, \mu=100 \mathrm{Pas}, \mathrm{g}=9.81 \mathrm{~m} / \mathrm{s}^{2}, \gamma=12$, and $\gamma_{c}=22.77$

Note that $Q$ is no longer the flow rate. Four cases emerge with (a) $c>0, Q=0$, (b) $c<0, Q>0$, (c) $c>0, Q<0$, and (d) $c>0, Q>0$. We do not consider (sub)cases in which $b$ may become zero and $Q \neq 0$. The cases (b), (c), and (d) with $\alpha>0$ correspond to the cases (b') $c>0, Q<0$, (c') $c<0, Q>0$, and (d') $c<0, Q<0$ for constant $\alpha<0$ when we reverse the sign of $b^{\prime}$. Hence, we do not consider cases with $\alpha<0$ separately.

For choked flow at the top boundary, the boundary condition for traveling waves is $Q=\gamma_{c} b_{T}{ }^{3 / 2}-$ $c b_{T}$. So for the traveling-wave free-boundary solutions for which we argued $Q=0$, this choked-flow condition gives $c=\gamma_{c} \sqrt{b_{T}}>0$.

Solutions with fronts exist for $Q \neq 0$ with finite discharge and infinite velocity at the front. The exception is the case $Q=0$ for which the velocity at the front is finite and the exact solution is

$$
\frac{\beta}{\alpha}[b-\sqrt{c / \alpha} \operatorname{arctanh}(\sqrt{\alpha} b / \sqrt{c})]=z-z_{r}(t)=z-z_{r 0}-c t
$$

with integration constant $z_{r 0}$ the position of the front at $t=0$.

The profiles for the depth, $z$, versus the aperture, $b(z, t)$, for the traveling-wave cases are shown in Fig. 4.7 for cases (a) $b^{\prime}<0$ and $Q=0$; (b) $b^{\prime}>0$; (c1) $b^{\prime}<0, b_{0}<b<b_{1}$ and (c2) $b^{\prime}>0, b>b_{1}$; and 

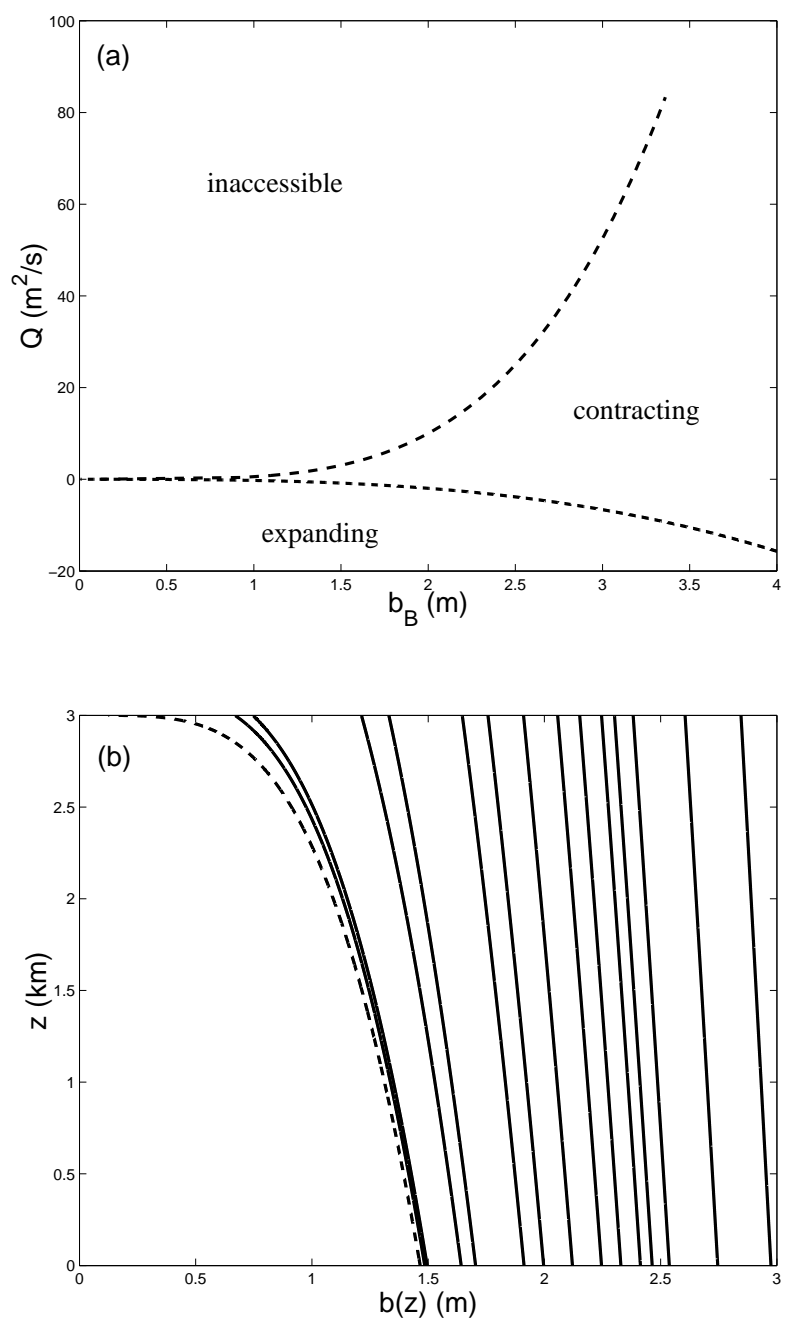

Fig. 4.3. Stationary case $\alpha<0$. (a) The parameter plane $Q-b_{B}$ with $b_{B}>0$ is from left to right divided into an inaccessible, contracting and expanding region. (b) Profiles of the vertical coordinate, $z$, versus the aperture, $b(z)$, of the relevant contracting region are displayed. The profile with the dashed line indicates the profile with critical speed at the top for which $u=\gamma_{c} \sqrt{b}$ and here $b=b_{T}=0.1245 \mathrm{~m}$ and $Q=2.7778 \mathrm{~m}^{2} / \mathrm{s}$. The parameters are $\alpha=-0.0883, H_{0}=3,000 \mathrm{~m}, D_{0}=1 \mathrm{~m}, \rho_{m}=2,500 \mathrm{~kg} / \mathrm{m}^{3}, \rho_{r}=2,600 \mathrm{~kg} / \mathrm{m}^{3}, \kappa=0.95, \lambda=10^{-7} \mathrm{~m}^{2} \mathrm{~s}^{2} / \mathrm{kg}, \mu=$ 100 Pas, $g=9.81 \mathrm{~m} / \mathrm{s}^{2}, \gamma=12$, and $\gamma_{c}=22.77$.

(d) $b^{\prime}>0$, corresponding to the cases in Fig. 4.6. The arrows indicate the direction or time propagation of the wave, and the dashed lines indicate the constant aperture limits corresponding to the roots in the cases (b) $b_{*}$, (c) $b_{0}, b_{1}$ with $b_{0}<b_{1}$, and (d) $b_{*}$.

\subsection{Numerical Verification}

To verify the algorithm and numerical implementation, a comparison is made between the numerical solutions of the partial differential equations with a mixture of (time-dependent) Dirichlet, choked-flow, and free-boundary conditions, and high(er) resolution exact or numerical solutions of the stationary and traveling-wave solutions governed by the relevant ordinary differential equation. For the full system, we use second-order accuracy in space and choose the time step sufficiently small, while, for the ordinary differential equations, we use a fourth-order Runge-Kutta spatial discretization and at least a tenfold higher resolution. Only the results of formulation F1 are shown because the results of the two formulations are similar. In each case, the total number of underlying fixed elements is stated. 

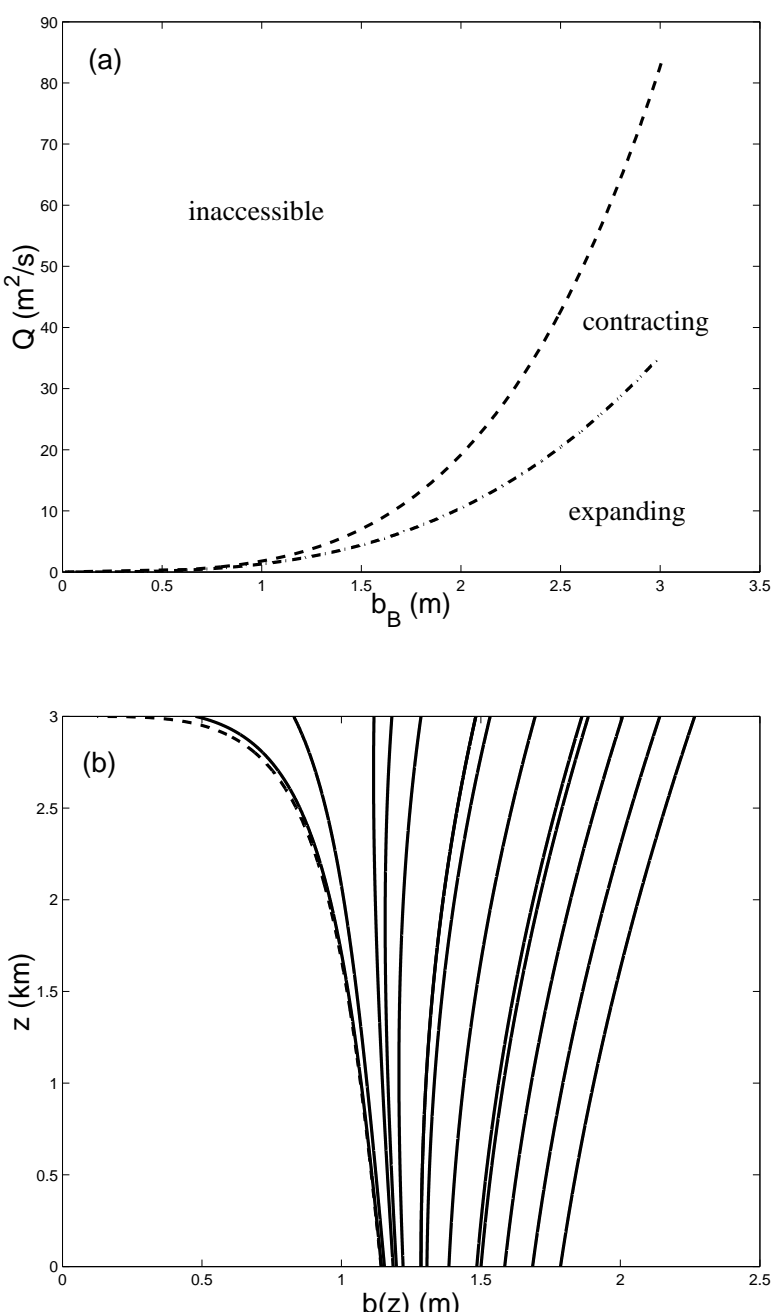

Fig. 4.4. Stationary case with $\alpha=\alpha(z)$. (a) The parameter plane $Q-b_{B}$ with $b_{B}>0$ is from left to right divided into a choked, contracting and expanding region. (b) Profiles of the vertical coordinate, $z$, versus the aperture, $b(z)$, of the latter two regions are displayed. The profile with critical speed at the top corresponds with the dashed line and $b_{T}=0.1245 \mathrm{~m}$. Parameter values are $H_{0}=3,000 \mathrm{~m}, D_{0}=1 \mathrm{~m}, \rho_{m}=2,500 \mathrm{~kg} / \mathrm{m}^{3}, \rho_{0}=2,800 \mathrm{~kg} / \mathrm{m}^{3}, \rho_{H}=2,900 \mathrm{~kg} / \mathrm{m}^{3}, \kappa=0.95, \lambda=$ $10^{-7} \mathrm{~m}^{2} \mathrm{~s}^{2} / \mathrm{kg}, \mu=100 \mathrm{Pas}, \mathrm{g}=9.81 \mathrm{~m} / \mathrm{s}^{2}$, and $\gamma=12$.

The boundary conditions used in the five presented simulations are summarized in Table 4.1. When nothing is mentioned, the flux rule as it stands in (A.3) is implemented.

We considered a stationary solution and two traveling-wave solutions, corresponding to Fig. 4.2 for (a) $c=0, \alpha>0$; and Fig. 4.7 for (a) $c>0, Q=0$ with $b<b_{*}$ and (c) $c>0, Q<0$ with $b_{0}<b<b_{1}$.

The exact stationary solution with Dirichlet and choked-flow boundary conditions, displayed in Fig. $4.2 \mathrm{~b}$ with the dashed line, was considered first. The sequence of numerical solutions approaching this state are displayed in Fig. 4.8. In Appendix A.4, it is shown that the spatial accuracy of the steady state solution in Fig. 4.8 reduces to order 1. An exact and numerical traveling-wave solution is shown in Fig. 4.9. The order of convergence is approximately 1.5 (Appendix A.4).

The exact and numerical traveling-wave solution with a free boundary propagating in the domain is provided in Fig. 4.10. The numerical solution uses the free-boundary strategy explained in §3.6. The numerical solution corresponds relatively well with the exact solution. Oscillations in the speed of the frontal position shown in Fig. 4.11 are caused by the element-splitting procedure. In Fig. 4.12, we show a more realistic case in which the opening crack adjusts to the choked-flow boundary conditions at the Earth's surface. 

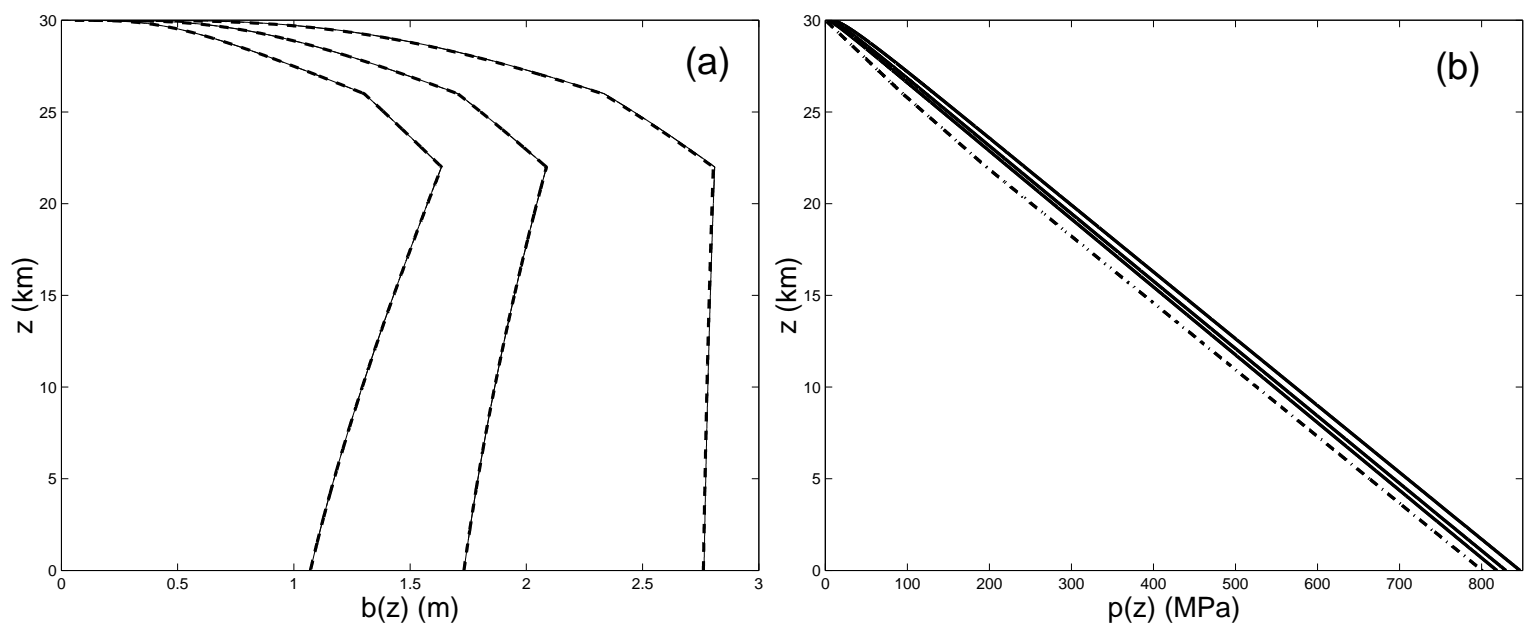

Fig. 4.5. Stationary solutions for the three flow rates $Q=b u=0.289,1.766,9.96 \mathrm{~m}^{2} / \mathrm{s}$ from left to right for both the elastic-flow and convection-diffusion models. (a) Depth versus width $b(z)$; note that these two solutions per flow rate are indistinguishable on the scale shown. (b) Depth versus the pressure of the three solutions (solid lines) and the lithostatic pressure (dashed line); note that the overpressures at the magma chamber lie between $18 M P a$ and $40 M P a$ and that the stress differences at depth are about $40 M P a$. Parameter values are assumed to model the Tolbachik volcano region in Kamchatka, Russia.
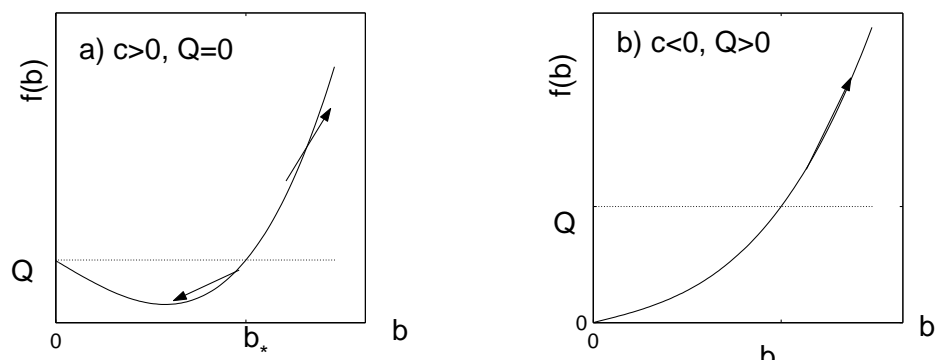

$b_{*}$
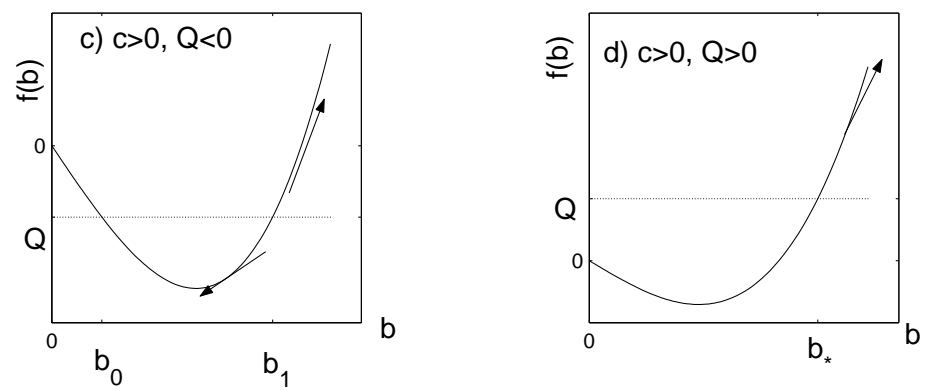

Fig. 4.6. $f(b)$ versus $b$ for the four traveling-wave cases considered with $\alpha>0$ : (a) for $c>0, Q=0$ there is one root $b_{*}$ and two situations $b<b_{*}$ where $b^{\prime}<0$, and $b>b_{*}$ where $b^{\prime}>0$; (b) for $c<0, Q>0$ there is one root $b_{*}$ and two situations $b<b_{*}$ where $b^{\prime}<0$, and $b>b_{*}$ where $b^{\prime}>0$; (c) for $c>0, Q<0$ there are two roots $b_{0}, b_{1}$ and three situations with $b<b_{0}$ where $b^{\prime}>0, b_{0}<b<b_{1}$ where $b^{\prime}<0$, and $b>b_{1}$ where $b^{\prime}>0$; and (d) for $c>0, Q>0$ there is one root $b_{*}$ and two situations $b<b_{*}$ where $b^{\prime}<0$, and $b>b_{*}$ where $b^{\prime}>0$. Arrows indicate whether $b^{\prime}>0$ or $b^{\prime}<0$ and are not added when $b \downarrow 0$ for $Q \neq 0$ as these cases are excluded. 
(a) $\mathrm{C}>0 \mathrm{Q}=0$

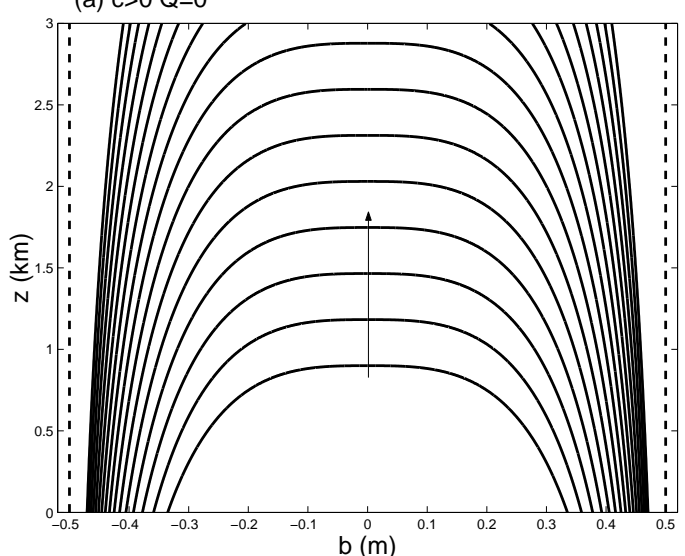

(c1) $c>0 Q<0$

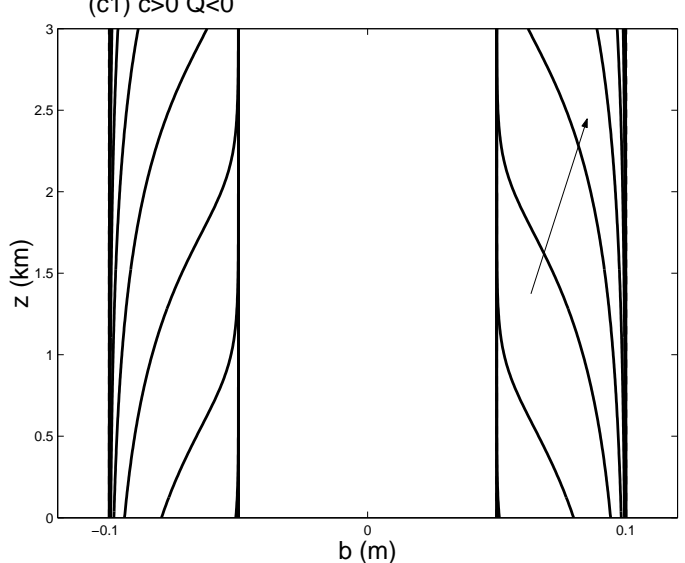

(d) $C>0 Q>0$

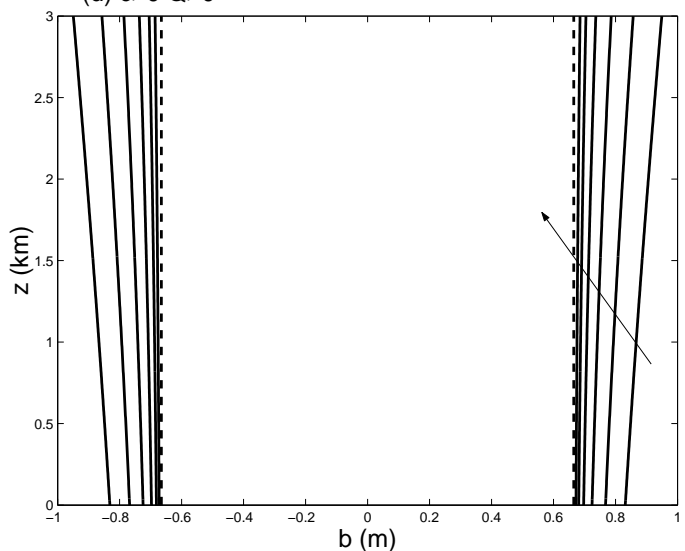

(b) $\mathrm{C}<0 \mathrm{Q}>0$

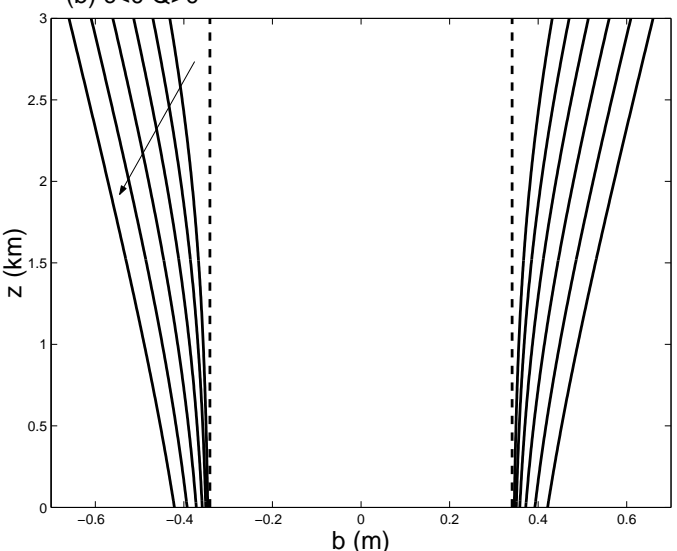

(c2) $c>0 Q<0$

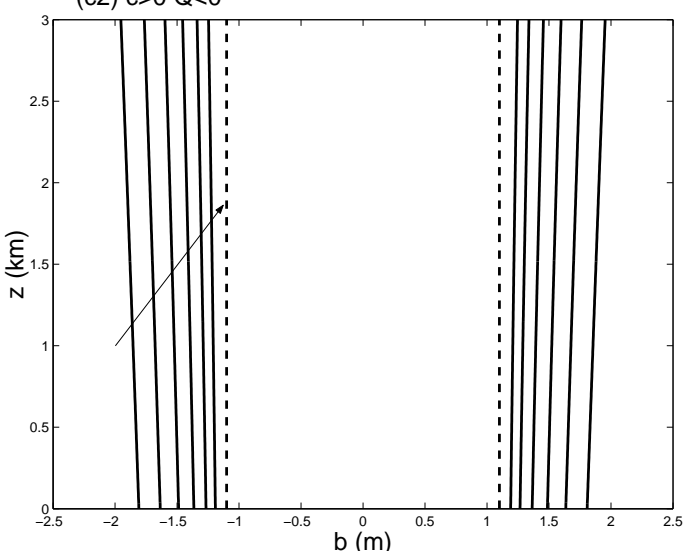

Fig. 4.7. Profiles of the depth, $z$, versus the aperture, $b(z, t)$, for the traveling-wave solutions with $\alpha>0$. Arrows indicate the propagation direction of the wave.

\subsection{Varying rock density and lava sinks}

The numerical model can handle the case with varying host rock density. In figure Fig. 4.13, an initially small and constant dike opening is adjusted to a larger chamber pressure. The time dependent solution with the choked-flow boundary conditions adjusts in 36 hours to one of the steady state solutions for the Kamchatka case described in Fig. 4.5. Note that $\alpha(z)$ changes sign at $z=26 \mathrm{~km}$.

Next, consider the flow in a stratified host rock from a magma chamber at $3 \mathrm{~km}$ depth to the surface with heavier magma relative to the local host rock, $\alpha<0$, for the last $1000 \mathrm{~m}$; and buoyant magma 


\begin{tabular}{c|c|c|c|c} 
Run & Location & Type & $\tilde{F}_{b}$ & $\tilde{F}_{q}$ \\
\hline \multirow{4}{*}{ Fig. 4.8 } & $z=0$ & Dirichlet & $\tilde{F}_{b}\left(b_{ \pm}, q_{+}\right)$ & $F_{q}\left(b_{-}\right)$ \\
& $z=H$ & choked & $\gamma_{c} b_{-}^{3 / 2}$ & $F_{q}\left(b_{-}\right)$ \\
Fig. 4.9 & $z=0$ & Dirichlet & $\tilde{F}_{b}\left(b_{ \pm}, q_{+}\right)$ & $F_{q}\left(b_{-}\right)$ \\
& $z=H$ & Dirichlet & $\tilde{F}_{b}\left(b_{ \pm}, q_{-}\right)$ & $F_{q}\left(b_{+}\right)$ \\
Fig. 4.10 & $z=0$ & Dirichlet & $\tilde{F}_{b}\left(b_{ \pm}, q_{+}\right)$ & $F_{q}\left(b_{-}\right)$ \\
& $z=z_{r}(t)$ & free & 0 & 0 \\
Fig. 4.12 & $z=H$ & Dirichlet & $\tilde{F}_{b}\left(b_{ \pm}, q_{-}\right)$ & $F_{q}\left(b_{+}\right)$ \\
& $z=0$ & Dirichlet & $\tilde{F}_{b}\left(b_{ \pm}, q_{+}\right)$ & $F_{q}\left(b_{-}\right)$ \\
& $z=z_{r}(t)$ & free & 0 & 0 \\
Fig. 4.13 & $z=H$ & choked & $\gamma_{c} b_{-}^{3 / 2}$ & $F_{q}\left(b_{-}\right)$ \\
& $z=0$ & Dirichlet & $\tilde{F}_{b}\left(b_{ \pm}, q_{+}\right)$ & $F_{q}\left(b_{-}\right)$ \\
& $z=H$ & choked & $\gamma_{c} b_{-}^{3 / 2}$ & $F_{q}\left(b_{-}\right)$ \\
& $z=0$ & Dirichlet & $\tilde{F}_{b}\left(b_{ \pm}, q_{+}\right)$ & $F_{q}\left(b_{-}\right)$ \\
Fig. 4.14.16 & $z=z_{r}(t)$ & free & 0 & 0 \\
& $z=H$ & choked & $\gamma_{c} b_{-}^{3 / 2}$ & $F_{q}\left(b_{-}\right)$ \\
& $z=0$ & Dirichlet & $\tilde{F}_{b}\left(b_{ \pm}, q_{+}\right)$ & $F_{q}\left(b_{-}\right)$ \\
& $z=z_{r}(t)$ & free & 0 & 0 \\
& $z=H$ & choked & $\gamma_{c} b_{-}^{3 / 2}$ & $F_{q}\left(b_{-}\right)$
\end{tabular}

Table 4.1. The boundary conditions are provided at $z=0$; and $z=z_{r}(t)$ (free boundary), $z=H$ (fixed) or $z=H(t)$ (dome growth), whichever is applicable, for the five runs presented for flux formulation F1 (A.3). The free-boundary conditions are valid provided $0<z_{r}(t)<H$.

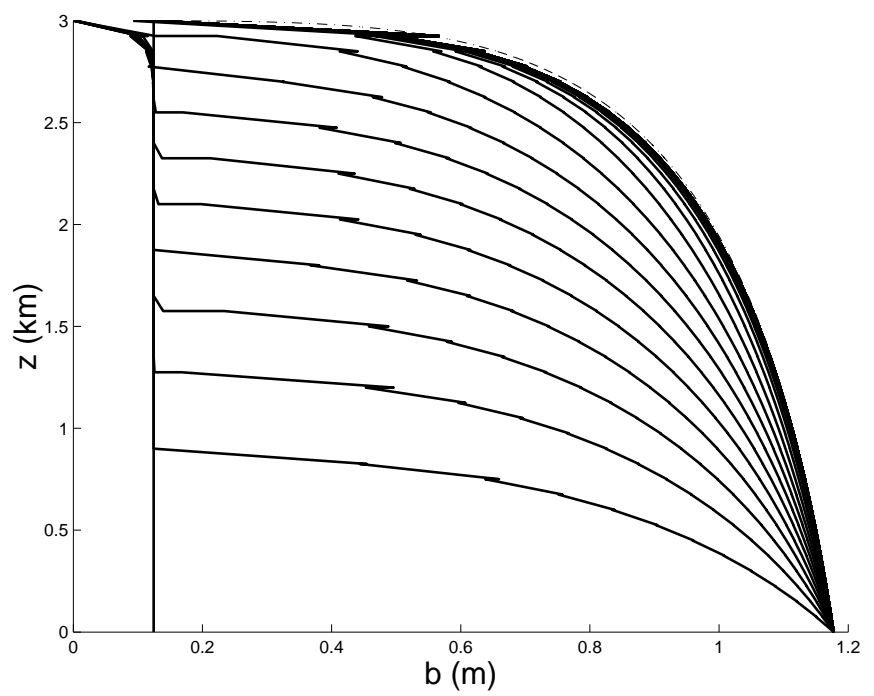

Fig. 4.8. The evolution of the aperture, $b(z, t)$, as function of depth, $z$, is shown for the numerical solution (solid lines) at times $0,1.8, \ldots, 45 \mathrm{~min}$ (dimensionless $t=0.1, \ldots, 2.5$ ). The final, nearly stationary state as a high-resolution (fourth order Runge-Kutta spatial discretization and 10 times higher resolution) numerical solution of the relevant ordinary differential equation is displayed as a dashed line. The Dirichlet condition at $z=0$ and initial conditions are $b_{B}=1.1774489332$ and $b(z, 0)=0.1244834652$. At the top, the choked flow is imposed. Other values are: $\alpha=0.4709$, $\beta=1.0$, and $\gamma_{c}=22.7683991532$. Forty equidistant elements are used.

relative to the host rock, $\alpha>0$, below a depth of $1000 \mathrm{~m}$. In Fig. 4.14(a), initially transient flow in a growing dike reaches the surface and evolves to an equilibrium roughly within the $64.8 \mathrm{~min}$. Thereafter, a periodic array of sinks of finite size opens along the magma dike at a depth of $z_{c}=2700 \mathrm{~m}(300 \mathrm{~m}$ below the surface). We assume here for simplicity that the sink diverts magma away continuously. In the case of drifts of finite length, not considered here, the diverted volume of magma would be finite, and hence the sinks would close in a finite time. We assume the sinks are close enough together, say $L_{t}=80 \mathrm{~m}$, to be treated as a line sink such that the non-uniformity in the lateral direction is 


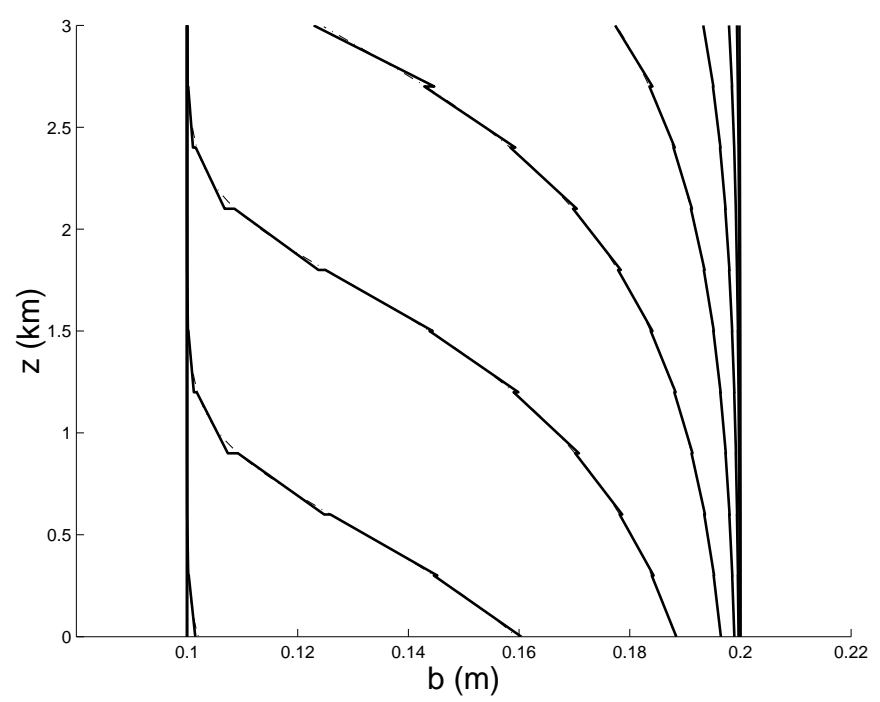

Fig. 4.9. The evolution of the aperture, $b(z, t)$, as function of depth, $z$, for the numerical solution (solid lines) at times $0,3.6, \ldots, 36 \mathrm{hr}$ (dimensionless $t=0,0.012, \ldots, 0.12$ ). The profile in the middle of the graph is used to determine the $L_{2}-$ and $L_{\infty}$-errors. The traveling-wave solution is a high-resolution (fourth order Runge-Kutta spatial discretization and approximately 10 times higher resolution) numerical solution of the relevant ordinary differential equation. For each time, this solution is displayed as a dashed line. The Dirichlet conditions are time dependent and given by the traveling-wave solution with dimensionless $c=32.9616, Q=-28.2528, \alpha=4.7088$ and $\beta=1.0$. Ten equidistant elements are used.

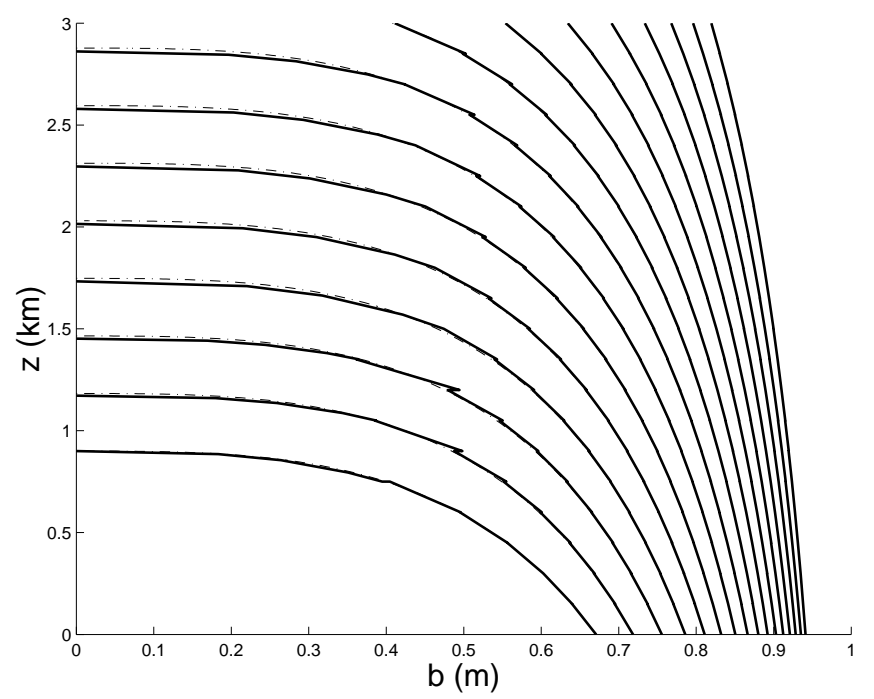

Fig. 4.10. The evolution of the aperture, $b(z, t)$, as function of depth, $z$, for the numerical solution (solid lines) at times $0,3.6, \ldots, 54 \mathrm{~min}$ (dimensionless $t=0,0.2, \ldots, 3$ ). The fifth profile at $14.4 \mathrm{~min}$ in the middle of the picture is used to determine the $L_{2}$ - and $L_{\infty}$-errors. The traveling-wave solution is provided by the exact solution for $Q=0$ (dashed lines). The Dirichlet conditions are time dependent and provided by the traveling-wave solution with dimensionless $c=\alpha=0.4709, \beta=1$. Twenty equidistant underlying regular elements are used, of which only a fraction is used with one time-dependent free-boundary element when the aperture is only open, $b>0$, in part of the domain.

minimal. Each separate sink has a lateral width of $L_{c}=5 \mathrm{~m}$ and a height of $h_{c}=5 \mathrm{~m}$, say. If the actual speed into a sink is $U_{f}$, then the effective speed into a sink along a corresponding "line sink" is $U_{d}=L_{c} U_{f} / L_{t}$. Hence, the one-dimensional continuity equation is still approximately valid

$$
\partial_{t} b+\partial_{z}(b u)=\left\{\begin{array}{cc}
0 & z<z_{c}-h_{c} / 2 \text { and } z>z_{c}+h_{c} / 2 \\
-Q_{s} / h_{c} & z_{c}-h_{c} / 2 \leq z \leq z_{c}+h_{c} / 2
\end{array}\right.
$$




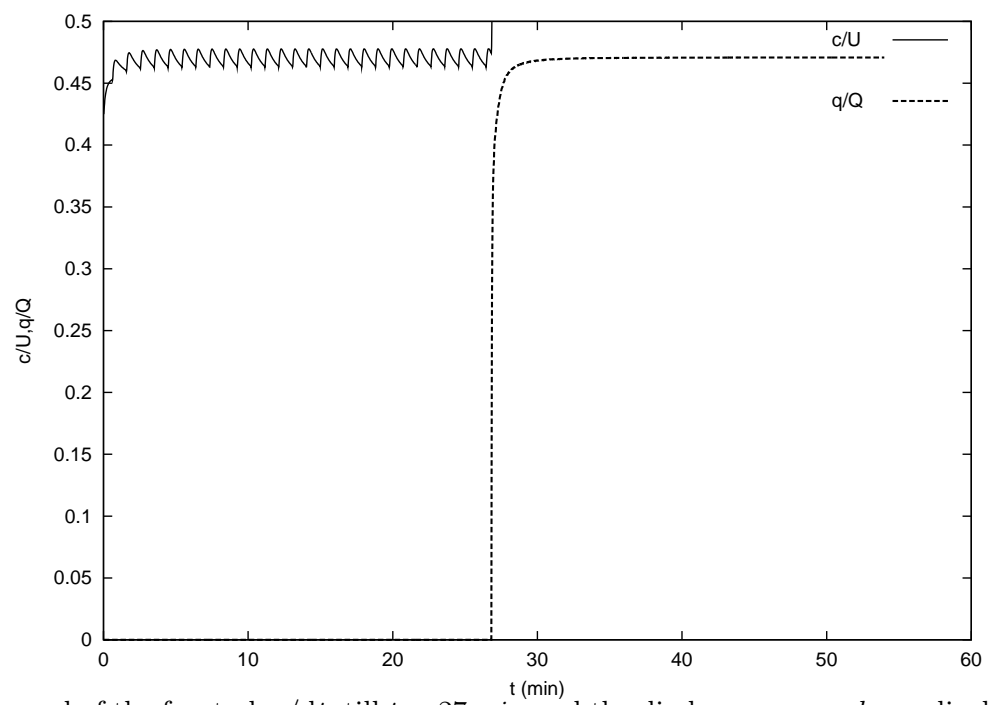

Fig. 4.11. The speed of the front, $\mathrm{d} z_{r} / \mathrm{d} t$, till $t \approx 27 \mathrm{~min}$ and the discharge, $q=u d$, are displayed at the end of the dike after $t \approx 27 \mathrm{~min}$. This speed should be $c / U=0.4709$ (with dimensional $U=2.778 \mathrm{~m} / \mathrm{s}$ ) and the discharge $q / Q=0.4709$ $\left(Q=2.778 \mathrm{~m}^{2} / \mathrm{s}\right)$. The oscillations in the frontal speed clearly demarcate the element-splitting process; there are 20 fixed elements.

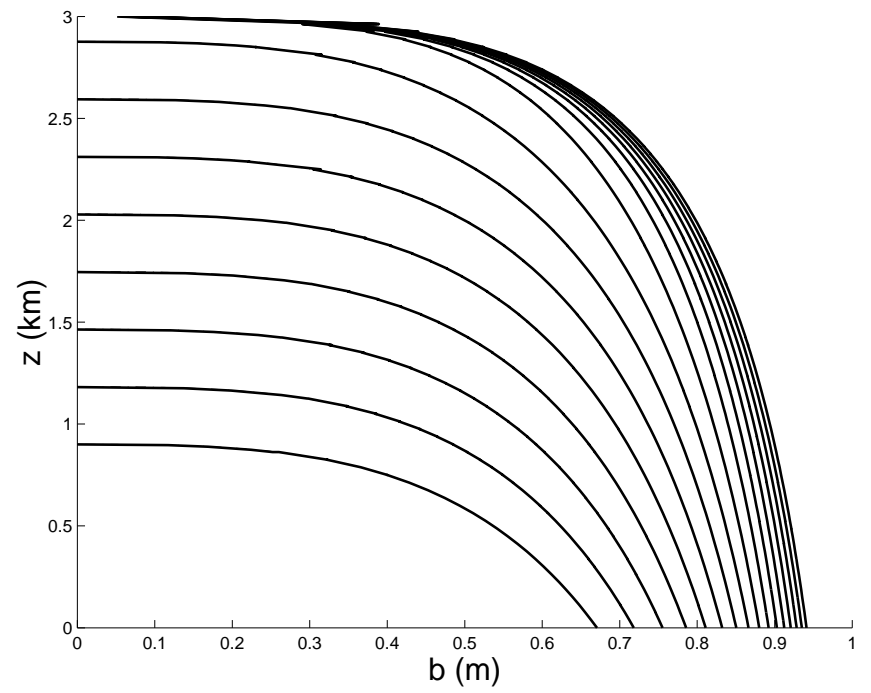

Fig. 4.12. The same simulation as in Fig. 4.10, but after the magma reaches the exit the flow at the exit is choked. Profiles at times $0,3.6 \mathrm{~min}, \ldots, 54 \mathrm{~min}$ (dimensionless $t=0,0.2, \ldots, 3$ ). The dashed lines represent the traveling-wave solution with corresponding exact solution before the magma meets the exit after $t=28.8 \mathrm{~min}$. This exact solution is used to determine the Dirichlet condition at $z=0$ for all times. There are 80 fixed elements.

with $Q_{s} / h_{c}=U_{d}$ for the dimensional case and $Q_{s} / h_{c}=U_{d} / \delta_{r}$ for the dimensionless case with $\delta_{r}=$ $D_{0} / H_{0} \ll 1$ (scaling $U_{d}$ with $U_{0}$ ). If we integrate $(4.7)$ in the dimensionless case across the source region over an interval larger than $h_{c}$, we can alternatively view the continuity equation with a sink $Q_{s} \delta\left(z-z_{c}\right)$ expressed with a delta function. So in dimensionless terms, the simplified continuity equation becomes $\partial_{t} b+\partial_{z}(b u)=-Q_{s} \delta\left(z-z_{c}\right)$. We used a dimensionless $Q_{s}=0.5$ in the simulation in Fig. 4.14(b), which amounts to $U_{f}=2.22 \mathrm{~m} / \mathrm{s}$ for the values provided in the text. Note how the array of sinks diverts magma away from the dike. While the sinks are diverting magma, reduction of the flow in the dike results in a small narrowing of the dike aperture (Fig. 4.14(b)). For larger values of $Q_{s}$, the magma dike may also close locally near the surface in response to the reduced magma pressure. For 


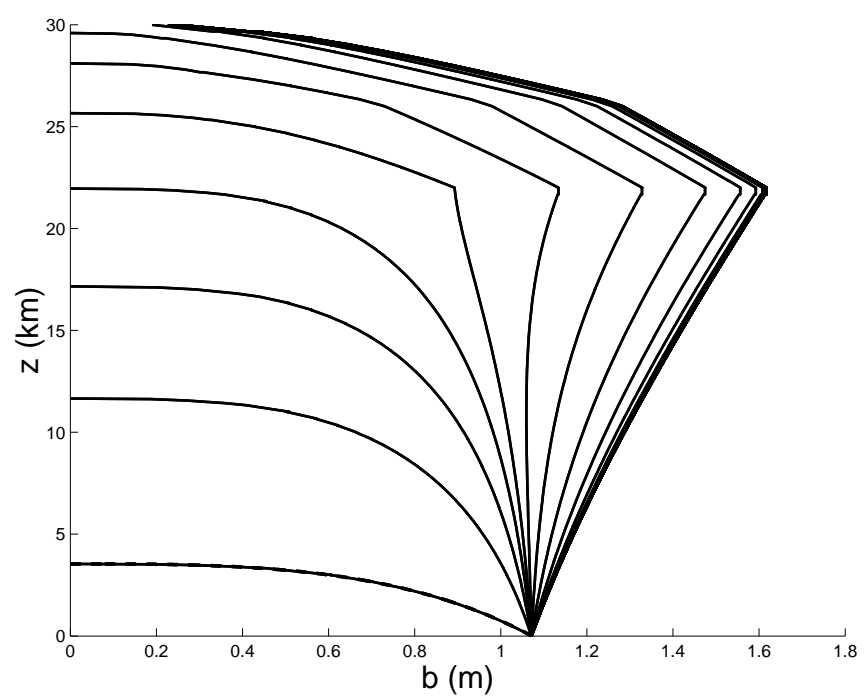

Fig. 4.13. The $36 \mathrm{hrs}$ of time evolution to one of the steady state solutions in Fig. 4.5 with $Q=0.289 \mathrm{~m}^{2} / \mathrm{s}$ and $\kappa=0.95$ for values based on the Tolbachik volcano region, Kamchatka.

(a)

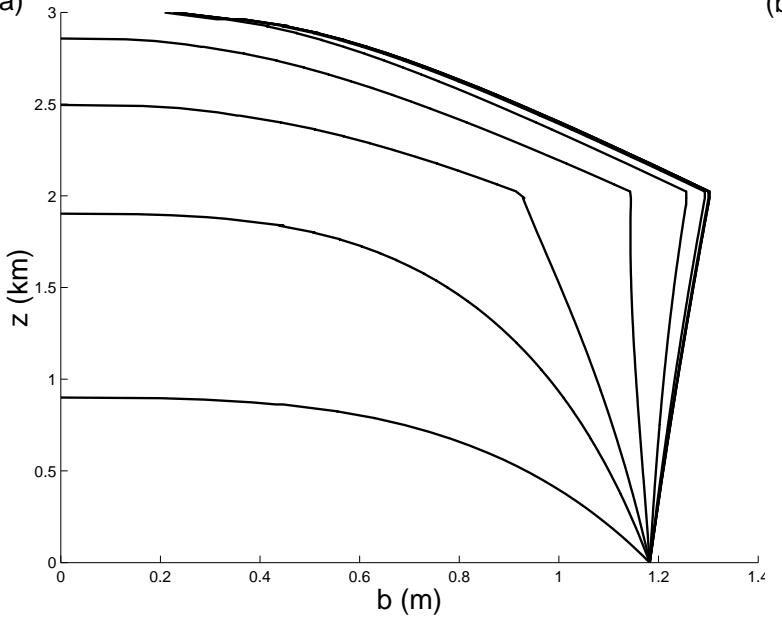

(b)

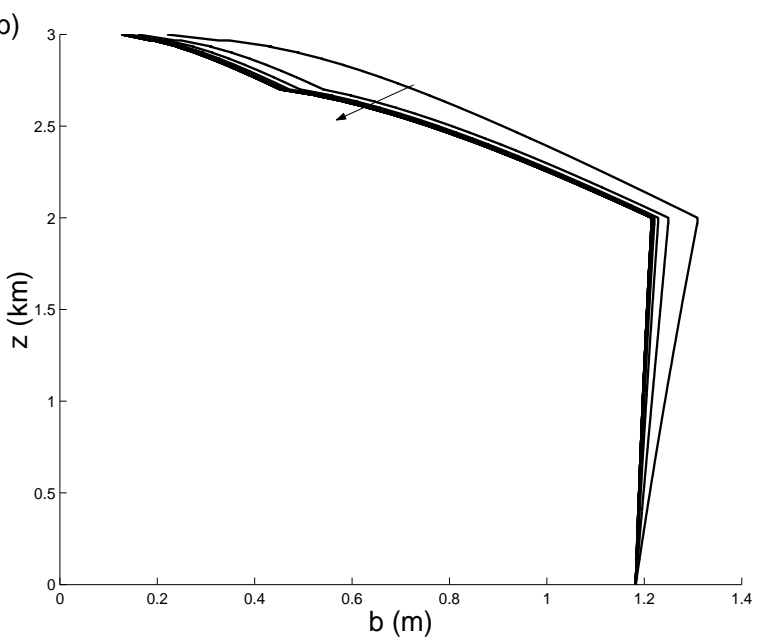

Fig. 4.14. (a) Time evolution to a steady state for $t<64.8 \min$ (dimensionless time $t=1.8$ ) with profiles displayed every $7.2 \mathrm{~min}$. (b) Time evolution from $64.8 \mathrm{~min} \leq t \leq 3 \mathrm{hrs}$ (dimensionless time $t=5$ ) with profiles displayed every $7.2 \mathrm{~min}$, when an array of magma sinks is open at $300 \mathrm{~m}$ below the surface. Parameter values used are: $\rho_{m}=2600 \mathrm{~kg} / \mathrm{m}^{3}, \rho_{r}=$ $2300 \mathrm{~kg} / \mathrm{m}^{3}$ for $z<2 \mathrm{~km}, \rho_{r}=2350 \mathrm{~kg} / \mathrm{m}^{3}$ for $z \geq 2 \mathrm{~km}, \lambda=2 \times 10^{-7} \mathrm{~m}^{2} \mathrm{~s}^{2} / \mathrm{kg}, \mu=100 \mathrm{Pas}$. The dimensionless $Q_{s}=0.5$, which amounts to $U_{f}=2.22 \mathrm{~m} / \mathrm{s}$ for the values provided in the text.

illustrative purposes, we used a small local value of the density of the host rock, $\rho_{r}=2350 \mathrm{~kg} / \mathrm{m}^{3}$, to get a relatively small negative value of $\alpha(z)$.

\subsection{Lava dome growth and collapse}

Quasi-periodic eruptions can emerge by several mechanisms. The emergence of multiple stable and unstable steady states often leads to quasi-periodic behavior. This arises for example because the viscosity is a nonlinear function of crystal or volatile content (e.g., Wylie et al., 2000), as these change when the magma decompresses towards the Earth's surface. 


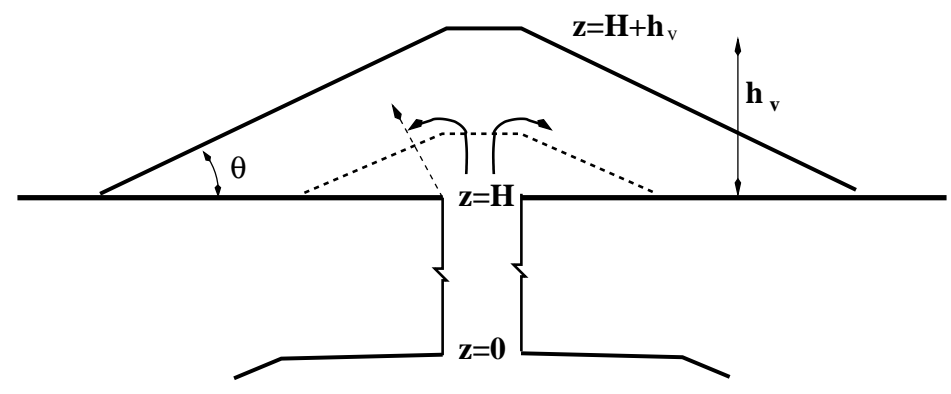

Fig. 4.15. The cross-sectional area of the lava dome is assumed to consist of the two triangles shown. The elongated dike is considered to have constant width as approximation to a very thin dike with an elliptical cross section in a horizontal plane. The dome height, $h_{v}(t)$, depends on the eruption area and the inclination angle, $\theta$.

The elastic advection-diffusion model yields another mechanism for quasi-periodic eruptions when a growing dome collapses every time it reaches certain critical conditions. Due to the dome growth the lithostatic pressure, $p_{r}$, increases. For a fixed chamber pressure $p_{B}$, the overpressure, $\Delta p_{B}$, in the magma chamber then decreases, as $\Delta p_{B}=p_{B}-p_{r}$. The lithostatic pressure $p_{r}$ follows by integrating (2.3), i.e., $\partial_{z} p_{r}=-\rho_{r} g$, from the magma chamber at $z=0$ to the exit at $H(t)=H(t=0)+h_{v}(t)$ with $h_{v}$ the height of the lava dome. Once the lava dome has reached a critical height, we assume that dome failure causes immediate removal of the dome on the slow time scale considered, such that the overpressure in the magma chamber is suddenly increased again.

We consider the growth of a lava dome of modest height as a consequence of a lava eruption at the Earth's surface. As long as the ratio of dome height, $h_{v}$, versus the depth of the magma chamber, $H(0)=10 \mathrm{~km}$, is small, the convection-diffusion equation is appropriate. Otherwise, the edifice load has to be taken into account, as in [5]. Parameter values taken are $\mu=1,000 \mathrm{~Pa} s, \lambda=10^{-7}, \kappa=0.95$, $\rho_{m}=2,500 \mathrm{~kg} / \mathrm{m}^{3}$, and constant $\rho_{r}=2,800 \mathrm{~kg} / \mathrm{m}^{3}$. The overpressure in the absence of a lava dome is approximately $10 \mathrm{MPa}$ against a lithostatic pressure of approximately $\rho_{r} g H(0)=275 \mathrm{MPa}$.

During its growth, a simplified shape of the dome, which cross-sectional area consists of two triangles, will be assumed. The shape of this dome is an approximation of a very elongated, solid elliptical cone excluding the volume of the dike through the cone, see Fig. 4.15. Hence, we deduce that, for a dome of height $h_{v}(t)$ and angle of incline $\theta$, the cross-sectional area, $O=O(t)$, is

$$
O(t)=h_{v}\left(w_{1 / 2}+h_{v} / \tan \theta\right) \approx h_{v}\left(b_{T} / 2+h_{v} / \tan \theta\right)
$$

with the half-width of the dike in the dome $w_{1 / 2} \approx b(H, t) / 2=b_{T}(t) / 2$. This area is the cumulation in time of the discharge of lava at the growing top of the dike and starts at time $t_{0}$ when the dike breaks through the surface at $z=H(0)$ with $H\left(t \leq t_{0}\right)=H(0)=10 \mathrm{~km}$ and $h_{v}\left(t_{0}\right)=0$. The width of the dike $b_{T}(t)=b(H, t)$ at the top of the cone is thus varying in time. The $w_{1 / 2}$-factor is included to avoid an infinite growth speed when $h_{v} \downarrow 0$. Alternatively, one could fill an area of fixed width so that the dome shape is rectangular. The shape of the dome is simply chosen to illustrate the leading-order dynamics and has no further significance. Lava flow will again start at some time less than $t_{0}$ from the magma chamber at $z=0$ following the exact solution (4.6). Upon breakthrough at time $t_{0}$ the speed $\mathrm{d} z_{r} / \mathrm{d} t$ will change abruptly from the speed of the lava front to the growth speed of the dome height, $\mathrm{d} h_{v} / \mathrm{d} t$

$$
\frac{\mathrm{d} h_{v}}{\mathrm{~d} t} \approx \frac{2 u_{T}(t) b_{T}(t)}{b_{T}+4 h_{v} / \tan \theta}
$$

with $h_{v}\left(t \leq t_{0}\right)=0$. This relationship follows from relating the area growth to the discharge rate at the top of the dike: $\mathrm{d} O(t) / \mathrm{d} t=u_{T}(t) b_{T}(t)$ with the critical velocity $u_{T}(t)=u(z=H, t)$ at the top, $z=H(t)$, of the dike and likewise for width $b_{T}$. In addition, we neglected $\partial b /\left.\partial t\right|_{z=H}$.

The bottom boundary condition is Dirichlet and follows from expression (4.6) minus the additional pressure increase because of the dome growth. After breakthrough, the width at the magma chamber decreases following the relation $b_{B}\left(t>t_{0}\right)=b_{B}\left(t_{0}\right)-\lambda \int_{H(0)}^{H} \rho_{r} g \mathrm{~d} z$ because the absolute pressure in the host rock increases as the dome grows in height. 


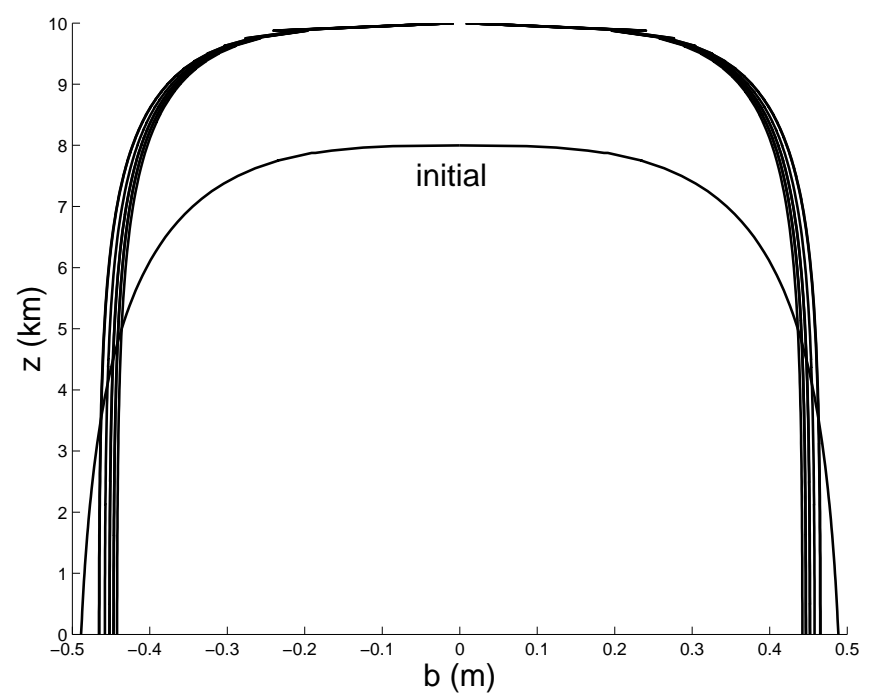

Fig. 4.16. Profiles of the evolution approximately every $67 \mathrm{hrs}$. After approximately five hours the dike reaches the surface, and the eruption reaches a periodic cycle. In total, 80 elements are used. The slope used is about $1: 20$.

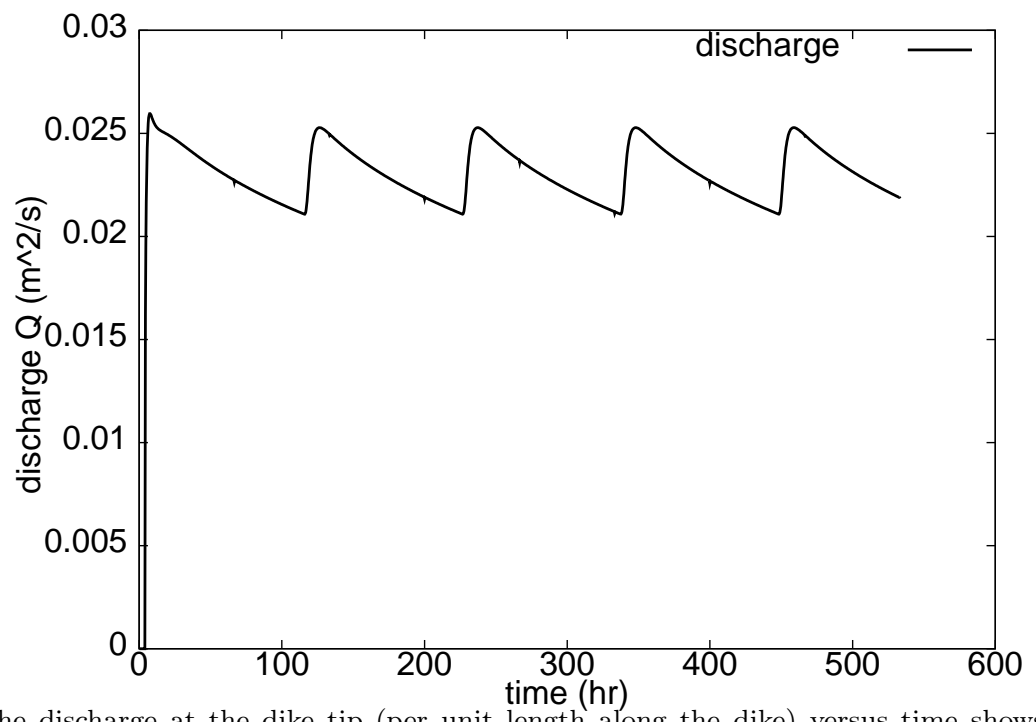

Fig. 4.17. The discharge at the dike tip (per unit length along the dike) versus time shows the eruption reaches a periodic cycle when the dome collapses every time it reaches a critical height, here chosen to be $h_{v}=50 \mathrm{~m}$.

After the dome growth starts, the dike width decreases as the constant magma overpressure needs to overcome a larger lithostatic pressure of the host rock and lava dome combined, see Fig. 4.16. In the initial phase, the dike propagates to the surface with a speed of $0.1308 \mathrm{~m} / \mathrm{s}$ to break through after approximately five hours. After breakthrough, the discharge in Fig. 4.17 becomes periodic as the dome collapses and disappears instantly (on the slow time scale modeled) after the dome height reaches a critical height chosen to be $h_{v}=50 \mathrm{~m}$. The periodicity observed is approximately $100 \mathrm{hrs}$.

\section{Conclusion and Discussion}

The flow of incompressible magma through a dike has been investigated in a model with leadingorder coupling between the elastic rock walls and the magma flow. The resulting convection-diffusion model admitted various stationary and traveling-wave solutions, and more complex numerical solutions were found with a new discretization method. The stationary and traveling-wave solutions were used as building blocks to understand the evolving flows and end-states in geophysical applications. More detailed information was obtained by performing simulations for a variety of time-dependent boundary 
conditions using the developed local discontinuous Galerkin finite-element method. The numerical method displayed a convergence of order 1.6 for smooth solutions in the $L_{2}$-norm also for a freeboundary solutions which were treated with a special arbitrary Lagrangian-Eulerian time-dependent element. For flows with steep gradients, the $L_{2}-$ norm reduced to order one because a slope limiter was needed to ensure stability (see, [2]). The $L_{\infty}$-error showed a convergence of 1.6 for a smooth solution without free boundary, and order 0.5 was reached in the free-boundary case and the case where steep gradients emerged. The more challenging discontinuous Galerkin method was chosen with an eye on extensions in two dimensions. However, in the one-dimensional case studied the finite element method proved crucial in the relatively accurate implementation of the nonlinear choked- flow and moving boundary conditions. We were as yet unable to successfully recover these results using a finite difference method (following, e.g., [4]).

The numerical finite-element method developed can readily be extended to asymmetric flows in the two-dimensional dike plane and can then be considered in complicated geometries, which may arise because of heterogeneities in the rock or dike intersection with a subsurface sink. For the twodimensional extension of the convection-diffusion model, it may be advisable to use an implicit CrankNicolson time discretization. The latter will permit longer time steps and enhance stability, in which case a slope limiter is probably no longer required $(c f .,[8])$.

The formulation of the proper in- and outflow boundary conditions in averaged models with a simplified elastic response of the host rock has remained an outstanding question. Nevertheless, the critical flow condition at the exit gave satisfactory results. In particular, steady-state solutions of the convection-diffusion model with the critical flow condition at the surface were nearly indistinguishable with steady-state solutions of the elastic model in which the inertia terms were not neglected. Solution of this elastic model with inertia, further geophysical applications of the current model for steady-state conditions and including the compressible effects of viscous, bubbly magma, are found in [11].

The convection-diffusion model can be extended to include more complex viscous behavior and volatiles. This could thus provide an interesting alternative to Wylie et al (1999), who considered an elastic response in the lower part of a conduit only (as opposed to the elongated dike considered here) but did include a nonlinear Arrhenius-type viscosity law representing viscosity changes due to volatile exsolution. Alternatively, a nonlinear viscosity law can arise due to temperature changes triggered by crystallization. Such nonlinear (viscous) behavior is known to yield multiple steady state solutions which in turn give rise to quasi-periodic (eruptive) solutions.

Presumably, improved conditions follow from further consideration of the feedback between the dynamics in the dike and the response of the magma chamber and the coupling with the erupted flows above and over the Earth's surface. Note that in [11], the dike width is in one case fixed at a depth proportional to $a$ (the major semi-axis of the ellipse) from the Earth surface where the choked-flow condition is imposed. This avoids the rapid changes in width at the Earth's surface but barely changes the width profiles at depth.

Acknowledgments: A. de Boer and O. Bokhove are grateful for the hospitality and support from the BP Institute for Multiphase Flow, Cambridge, U.K. O.B. acknowledges a fellowship from The Royal Dutch Academy of Arts and Sciences (KNAW), and support from the Center for Nuclear Waste Regulatory Analyses (CNWRA), Southwest Research Institute, San Antonio, Texas, U.S.A. We thank B.E. Hill (CNWRA) and O. Melnik for their scientific comments.

This paper was prepared to document work performed by the Center for Nuclear Waste Regulatory Analyses (CNWRA) for the Nuclear Regulatory Commission (NRC) under Contract No. NRC-02-02-012. The activities reported here were performed on behalf of the NRC Office of Nuclear Material Safety and Safeguards, Division of High-Level Waste Repository Safety. This paper is an independent product of the CNWRA and does not necessarily reflect the view or regulatory position of the NRC.

\section{References}

1. Bokhove, O.: Flooding and drying in discontinuous Galerkin finite-element discretizations of shallow-water equations. Part 1: one dimension. J. of Sci. Comp. 22, (2005)

2. Burbeau, A., Sagaut, P., and Bruneau, Ch.-H.: A problem-independent limiter for high-order Runge-Kutta Discontinuous Galerkin methods. J. Comp. Phys. 169, 111-150 (2001)

3. Cockburn, B., and Shu, C.-W.: The local discontinuous Galerkin method for time-dependent convection-diffusion systems. Siam. J. Numer. Anal. 135, 2240-2463 (1998) 
4. Morton, K.W., and Mayers, D.F.: Numerical solution of partial differential equations, Cambridge University Press. (1994)

5. Pinel, P., and Jaupart, C.: The effect of edifice load on magma ascent beneath a volcano. In: Causes and consequences of eruptions of andesite volcanoes. Eds. P. Francis, J. Neuberg and R.S.J. Sparks. Phil. Trans. R. Soc. London. A 358, 1515-1532 (2000)

6. Shu, C-W., and Osher, S.: Efficient implementation of essentially non-oscillatory shock-capturing schemes II. J. Comp. Phys. 83, 32-78 (1989)

7. Stock, J.M., Healy, J.H., Hickman, S.H., and Zoback, M.D.: Hydraulic fracturing stress measurements at Yucca Mountain, Nevada, and relationship to the regional stress field. Journal of Geophysical Research 90 (B10), 86918706 (1985).

8. Yan, J, and Shu, C.-W.: Local discontinuous Galerkin methods for partial differential equations with higher order derivatives. J. Sci. Comp. 17 27-47 (2002)

9. Whitham, G.B.: Linear and nonlinear waves, John Wiley, 636 pp. (1974)

10. Woods, A.W.: The dynamics of explosive volcanic eruptions. Rev. of Geophys. 33, 495-530. (1995)

11. Woods, A.W., Bokhove, O., Boer de, A., and Hill, B.E.: Compressible magma flow in a two-dimensional elasticwalled conduit. Under review for Earth and Planetary Sci. Lett., 18 pp. (2005)

12. Wylie, J.J., Voight, B, and Whitehead, J.A. Instability of magma flow from volatile-dependent viscosity. Science 285, 1883-1885 (1999)

\section{A Discontinuous Galerkin Finite-Element Method}

\section{A.1 Time integration}

We write (3.13) as a system of ordinary differential and algebraic equations

$$
\frac{\mathrm{d} \mathbf{b}}{\mathrm{d} t}=\mathbf{G}_{b}(\mathbf{b}, \mathbf{q}) \quad \text { and } \quad \mathbf{q}=\mathbf{G}_{q}(\mathbf{b})
$$

with $\mathbf{b}=(\bar{B}, \hat{B})^{T}$ the state vector of unknown coefficients of the basis functions, and $\mathbf{q}=(\bar{Q}, \hat{Q})^{T}$. We can then use the third-order Runge-Kutta method of [6], for example, to discretize (A.1) in time, and obtain

$$
\begin{aligned}
\mathbf{q}^{n}=\mathbf{G}_{q}\left(\mathbf{b}^{n}\right), & \mathbf{b}^{(1)}=\mathbf{b}^{n}+\Delta t \mathbf{G}_{b}\left(\mathbf{b}^{n}, \mathbf{q}^{n}\right) \\
\mathbf{q}^{(1)}=\mathbf{G}_{q}\left(\mathbf{b}^{(1)}\right), & \mathbf{b}^{(2)}=\left[3 \mathbf{b}^{n}+\mathbf{b}^{(1)}+\Delta t \mathbf{G}_{b}\left(\mathbf{b}^{(1)}, \mathbf{q}^{(1)}\right)\right] / 4 \\
\mathbf{q}^{(2)}=\mathbf{G}_{q}\left(\mathbf{b}^{(2)}\right), & \mathbf{b}^{n+1}=\left[\mathbf{b}^{n}+2 \mathbf{b}^{(2)}+2 \Delta t \mathbf{G}_{b}\left(\mathbf{b}^{(2)}, \mathbf{q}^{(2)}\right)\right] / 3 .
\end{aligned}
$$

Note that we can solve for $\mathbf{b}$ and $\mathbf{q}$ in an explicit manner because the new (intermediate) stage of $\mathbf{q}$ can always be found from the new (intermediate) stage of $\mathbf{b}$ before commencing the time update.

\section{A.2 Flux formulation}

The numerical flux, $\tilde{F}\left(w_{-}, w_{+}\right)$, is chosen to (i) be locally Lipschitz, following [3], implying that there is a constant, $K \geq 0$, such that

$$
\left|\tilde{F}\left(w_{-}, w_{+}\right)-F(\bar{u})\right| \leq K \max \left(\left|w_{-}-\bar{u}\right|,\left|w_{+}-\bar{u}\right|\right)
$$

for all $w_{-}, w_{+}$with $\left|w_{-}-\bar{u}\right|$ and $\left|w_{+}-\bar{u}\right|$ sufficiently small, (ii) be consistent such that $\tilde{F}(b, b)=F(b)$, (iii) ensure a local determination of $q_{h}$ in terms of $b_{h}$, (iv) reduce to an E-flux in the conservative limit when $\beta=0$, that is,

$$
\int_{b_{-}}^{b_{+}} F_{b}(s ; \beta=0)-\tilde{F}_{b}\left(b_{-}, b_{+} ; \beta=0\right) \mathrm{d} s \geq 0
$$

with $F_{b}(b ; \beta=0)=\alpha b^{3}$, and $(\mathrm{v})$ be $L_{2}$-stable, as will be shown. Note that the flux is the only way of communication between elements, and that the flux is determined by the values of $b_{h}$ and $q_{h}$ immediately left and right of each face.

To ensure positivity of the mean, an upwind scheme is chosen in contrast to the scheme developed by [3], which turned out to be less or unstable in our application. It is, therefore, necessary to reconsider their $L_{2}$-stability analysis. 
From such an analysis, the following numerical fluxes emerge for case F1 in (3.2)

$$
\begin{aligned}
& \tilde{F}_{b}=\tilde{F}_{b}\left(b_{ \pm}, q_{ \pm}\right)=\left\{\begin{array}{llll}
\frac{1}{2} \alpha\left(b_{+}^{2}+b_{-}^{2}\right) b_{-}+\beta q_{+} b_{-} \sqrt{b_{-}} \text {if } & \alpha>0 & \& & q_{+}>0 \\
\frac{1}{2} \alpha\left(b_{+}^{2}+b_{-}^{2}\right) b_{-}+\beta q_{+} b_{+} \sqrt{b_{+}} \text {if } & \alpha>0 & \& & q_{+}<0 \\
\frac{1}{2} \alpha\left(b_{+}^{2}+b_{-}^{2}\right) b_{+}+\beta q_{+} b_{-} \sqrt{b_{-}} \text {if } & \alpha<0 & \& & q_{+}>0 \\
\frac{1}{2} \alpha\left(b_{+}^{2}+b_{-}^{2}\right) b_{+}+\beta q_{+} b_{+} \sqrt{b_{+}} \text {if } & \alpha<0 & \& & q_{+}<0
\end{array}\right. \\
& \tilde{F}_{q}=F_{q}\left(b_{-}\right) .
\end{aligned}
$$

Alternatively, we also tested case F1'

$$
\begin{aligned}
& \tilde{F}_{b}=\tilde{F}_{b}\left(b_{ \pm}, q_{ \pm}\right)=\left\{\begin{array}{lllll}
\frac{1}{2} \alpha\left(b_{+}^{2}+b_{-}^{2}\right) b_{-}+\beta \bar{q} b_{-} \sqrt{b_{-}} \text {if } & \alpha>0 & \& & \bar{q}>0 \\
\frac{1}{2} \alpha\left(b_{+}^{2}+b_{-}^{2}\right) b_{-}+\beta \bar{q} b_{+} \sqrt{b_{+}} \text {if } & \alpha>0 & \& & \bar{q}<0 \\
\frac{1}{2} \alpha\left(b_{+}^{2}+b_{-}^{2}\right) b_{+}+\beta \bar{q} b_{-} \sqrt{b_{-}} \text {if } & \alpha<0 & \& & \bar{q}>0 \\
\frac{1}{2} \alpha\left(b_{+}^{2}+b_{-}^{2}\right) b_{+}+\beta \bar{q} b_{+} \sqrt{b_{+}} \text {if } & \alpha<0 & \& & \bar{q}<0
\end{array}\right. \\
& \tilde{F}_{q}=\tilde{F}_{q}\left(b_{ \pm}\right)=\bar{F}_{q}=\left[F_{q}\left(b_{-}\right)+F_{q}\left(b_{+}\right)\right] / 2 .
\end{aligned}
$$

For case F2 in (3.3), we choose

$$
\begin{aligned}
& \tilde{F}_{b}=\tilde{F}_{b}\left(b_{ \pm}, q_{ \pm}\right)=\left\{\begin{array}{lll}
\overline{u_{s}} b_{-}^{3 / 2} & \text { if } & \overline{u_{s}} \geq 0 \\
\overline{u_{s}} b_{+}^{3 / 2} & \text { if } & \overline{u_{s}}<0
\end{array}\right. \\
& \tilde{F}_{q}=\tilde{F}_{q}\left(b_{ \pm}\right)=\bar{F}_{q}=\left[F_{q}\left(b_{-}\right)+F_{q}\left(b_{+}\right)\right] / 2 \text {. }
\end{aligned}
$$

For case F1, we separately use an upwind scheme both for the "convective" wind, $u_{\text {conv }}=\alpha b^{2}$, and the "diffusive" wind component. For case F2, we use an upwind scheme based on the "scaled" velocity, $u_{s}=\alpha b^{3 / 2}+\beta q$, defined in (3.2). Inspection shows that these fluxes are locally Lipschitz and consistent. The diffusive part of the flux (A.3) for case F1 alternates which is reported to be more stable [8]. The local character follows from the spatial discretization in which $q$, knowing $b$ at the previous time level, can be solved before it is used in the discrete equations for the aperture, $b$; see also section A.1.

Properties (iv) and (v) are proven next. The flux F2 is more compact than flux F1. Readers less interested in further numerical analysis could proceed to the numerics to ensure positivity of the approach and the treatment of the free boundary in $\S 3.5$ and further, or to the results in $\S 4$.

\section{A.3 $L_{2}$-stability}

$L_{2}$-stability for the discretized equations follows in an analogy of the $L_{2}$-stability for the continuous case with $\alpha$ constant. We simply assume that for general $\alpha(z)$ the resulting discretization holds as well. In addition, the case without free boundary is analyzed. To wit, multiply (3.1) with $b$ and $q$, respectively, sum, and integrate over space and time to obtain:

$$
\begin{gathered}
\frac{1}{2} \int_{0}^{H} b^{2}-b_{i}^{2} \mathrm{~d} z+\int_{0}^{T} \int_{0}^{H} \beta q^{2} \mathrm{~d} z \mathrm{~d} t-\int_{0}^{T} \int_{0}^{H}\left(F_{b} \partial_{z} b+F_{q} \partial_{z} q\right) \mathrm{d} z \mathrm{~d} t+ \\
\int_{0}^{T}\left(u b^{2}+F_{q} q\right)_{z=H}-\left(u b^{2}+F_{q} q\right)_{z=0} \mathrm{~d} t=0 \Longleftrightarrow \\
\frac{1}{2} \int_{0}^{H} b^{2}-b_{i}^{2} \mathrm{~d} z+\int_{0}^{T} \int_{0}^{H} \beta q^{2} \mathrm{~d} z \mathrm{~d} t+ \\
\int_{0}^{T}\left(u b^{2}-\alpha b^{4} / 4\right)_{z=H}-\left(u b^{2}-\alpha b^{4} / 4\right)_{z=0} \mathrm{~d} t=0
\end{gathered}
$$

because

$$
F_{b} \partial_{z} b+F_{q} \partial_{z} q=\alpha b^{3} \partial_{z} b+\beta q b^{3 / 2} \partial_{z} b+(2 / 5) \beta b^{5 / 2} \partial_{z} q=\partial_{z}\left[\phi(b)+F_{q} q\right]
$$

with $\phi(b)=\int_{0}^{b} \alpha s^{3} \mathrm{~d} s=\alpha b^{4} / 4$. Note that $b_{i}(z)=b(z, 0)$. 
The discrete version of $L_{2}$-stability for flux cases F1 and F2 proceeds as follows (cf., [3]); by adding the weak formulation (3.7) of both equations, we find

$$
\begin{aligned}
\mathcal{B}_{h}\left(w_{h}, v_{h}\right)= & \int_{0}^{T} \int_{0}^{H} \frac{\mathrm{d} b_{h}}{\mathrm{~d} t} v_{b}+\beta v_{q} q_{h} \mathrm{~d} z \mathrm{~d} t-\int_{0}^{T} \sum_{2 \leq k \leq N_{e}}\left(\tilde{F} \cdot\left[\left[v_{h}\right]\right]\right)_{k} \mathrm{~d} t+ \\
& \int_{0}^{T}\left(\tilde{F} \cdot v_{h}^{-}\right)_{k=N_{e}+1}-\left(\tilde{F} \cdot v_{h}^{+}\right)_{k=1} \mathrm{~d} t-\int_{0}^{T} \sum_{1 \leq k \leq N_{e}} \int_{K_{k}} F \cdot \partial_{z} v_{h} \mathrm{~d} z \mathrm{~d} t
\end{aligned}
$$

with $w_{h}=\left(b_{h}, q_{h}\right), v_{h}=\left(v_{b}, v_{q}\right)$, and $[[v]]=v_{+}-v_{-}$. As in the continuous case, substitute $v_{b}=b_{h}$ and $v_{q}=q_{h}$ into (A.7) to obtain:

where

$$
\begin{aligned}
\mathcal{B}_{h}\left(w_{h}, w_{h}\right) & =\frac{1}{2} \int_{0}^{H}\left(b_{h}^{2}-b_{h i}^{2}\right) \mathrm{d} z+\int_{0}^{T} \int_{0}^{H} \beta q_{h}^{2} \mathrm{~d} z \mathrm{~d} t+ \\
& \int_{0}^{T}\left(\phi\left(b_{h}^{+}\right)+F_{q}^{+} q_{h}^{+}-\tilde{F} \cdot w_{h}^{+}\right)_{k=1} \mathrm{~d} t- \\
& \int_{0}^{T}\left(\phi\left(b_{h}^{-}\right)+F_{q}^{-} q_{h}^{-}-\tilde{F} \cdot w_{h}^{-}\right)_{k=N_{e}+1} \mathrm{~d} t+\int_{0}^{T} \Theta_{\text {dissipation }}(t) \mathrm{d} t
\end{aligned}
$$

Rewriting

$$
\begin{gathered}
\Theta_{\text {dissipation }}(t)=-\sum_{2 \leq k \leq N_{e}} \tilde{F} \cdot\left[\left[w_{h}\right]\right]-\sum_{1 \leq k \leq N_{e}} \int_{K_{k}} F \cdot \partial_{z} w_{h} \mathrm{~d} z+ \\
{\left[\phi\left(b_{h}\right)+F_{q} q_{h}\right]_{k=N_{e}+1}^{-}-\left[\phi\left(b_{h}\right)+F_{q} q_{h}\right]_{k=1}^{+}}
\end{gathered}
$$

is used to evaluate (A.9) further. Hence, requiring that

$$
\begin{aligned}
\Theta_{\text {dissipation }}(t) & =\sum_{2 \leq k \leq N_{e}} \Theta_{\text {dissipation }}^{k}(t)=\sum_{2 \leq k \leq N_{e}}\left\{\left[\left[\phi\left(b_{h}\right)+F_{q} q_{h}\right]\right]-\tilde{F} \cdot\left[\left[w_{h}\right]\right]\right\}_{k} \\
& =\sum_{2 \leq k \leq N_{e}}\left\{\left[\left[\phi\left(b_{h}\right)\right]\right]+\left[\left[F_{q}\right]\right] \bar{q}_{h}-\left[\left[b_{h}\right]\right] \tilde{F}_{b}+\bar{F}_{q}\left[\left[q_{h}\right]\right]-\left[\left[q_{h}\right]\right] \tilde{F}_{q}\right\}_{k}>0
\end{aligned}
$$

motivates the choice (A.3) and its limits. Reordering the chosen convective flux (3.2) in the limit $\beta=0$ produces

$$
\begin{aligned}
\lim _{\beta \rightarrow 0} \Theta_{\text {dissipation }}^{k}(t) & =\left[\left[\phi\left(b_{h}\right)\right]\right]-\left[\left[b_{h}\right]\right] \frac{1}{2} \alpha\left(b_{-}^{2}+b_{+}^{2}\right) b_{ \pm} \\
& =\int_{b_{-}}^{b_{+}} F_{b}(s ; \beta=0)-\frac{1}{2} \alpha\left(b_{-}^{2}+b_{+}^{2}\right) b_{ \pm} \mathrm{d} s>0
\end{aligned}
$$

for $F_{b}(b ; \beta=0)=\alpha b^{3}$, when $\alpha \neq 0$ proving property (iv), that the convective part of the flux is an E-flux. The flux, $\tilde{F}_{b}$, in the diffusive limit is also an upwind flux as it introduces extra stabilizing dissipation because some (graphical) analysis shows that

$$
-\left[\left[q_{h}\right]\right] F_{q}\left(b_{-}\right)+\left[\left[F_{q}\right]\right] \bar{q}_{h}-\beta\left[\left[b_{h}\right]\right] q_{+}\left\{\begin{array}{lll}
\sqrt{b_{-}} b_{-} & \text {if } & q_{+} \geq 0 \\
\sqrt{b_{+}} b_{+} & \text {if } & q_{+}<0
\end{array}\right\}>0 .
$$

For the flux F2, we have been unable to prove $L_{2}$-stability when $\alpha \neq 0$ and $\beta \neq 0$ because the convective and diffusive parts in general cannot be considered in separation. Nevertheless, in the frictionless limit, $\beta \rightarrow 0$, we find

$$
\begin{aligned}
\lim _{\beta \rightarrow 0} \Theta_{\text {dissipation }}^{k}(t) & =\left[\left[\phi\left(b_{h}\right)\right]\right]-\left[\left[b_{h}\right]\right] \frac{1}{2} \alpha\left(b_{-}^{3 / 2}+b_{+}^{3 / 2}\right) b_{ \pm}^{3 / 2} \\
& =\int_{b_{-}}^{b_{+}} F_{b}(s ; \beta=0)-\frac{1}{2} \alpha\left(b_{-}^{3 / 2}+b_{+}^{3 / 2}\right) b_{ \pm}^{3 / 2} \mathrm{~d} s>0
\end{aligned}
$$

while in the diffusive limit, $\alpha \rightarrow 0$, a similar analysis as in (A.13) holds again. 


\begin{tabular}{c|c|c|c|c|c|c|c}
$N=20$ & $\begin{array}{c}N=40 \\
\text { Error }\end{array}$ & Error & Order & $\begin{array}{c}N=80 \\
\text { Error }\end{array}$ & Order & $\begin{array}{c}N=160 \\
\text { Error }\end{array}$ & Order \\
\hline$L_{2}$ & 0.0376041 & 0.020769 & 0.86 & 0.011052 & 0.91 & 0.005590 & 0.98 \\
$L_{\infty}$ & 0.037604 & 0.16191 & 0.38 & 0.12085 & 0.42 & 0.08668 & 0.48
\end{tabular}

Table A.1. $L_{2}$ and $L_{\infty}$-errors as function of resolution and the spatial order of accuracy for the stationary case with $c=0, \alpha>0$. Polynomial order $d_{P}=1$. Solutions are considered at dimensionless time $t=2.5$.

\begin{tabular}{c|c|c|c|c|c|c|c} 
& $\begin{array}{c}N=10 \\
\text { Error }\end{array}$ & $\begin{array}{c}N=20 \\
\text { Error }\end{array}$ & Order & $\begin{array}{c}N=40 \\
\text { Error }\end{array}$ & Order & $\begin{array}{c}N=80 \\
\text { Error }\end{array}$ & Order \\
\hline$L_{2}$ & 0.0067878 & 0.0022502 & 1.5929 & 0.0007432 & 1.5982 & 0.0002381 & 1.6419 \\
$L_{\infty}$ & 0.0140886 & 0.0049057 & 1.5220 & 0.0016545 & 1.5680 & 0.0005139 & 1.6870
\end{tabular}

Table A.2. $L_{2}$ - and $L_{\infty}$-errors as functions of resolution and the spatial order of accuracy for the traveling-wave case with $c>0, Q<0, b_{0}<b<b_{1}$. Polynomial order $d_{P}=1$. Solutions are considered at dimensionless time $t=0.06$.

\begin{tabular}{c|c|c|c|c|c|c|c|c|c}
$N=10$ & $\begin{array}{c}N=20 \\
\frac{\text { Error }}{10^{-4}}\end{array}$ & $\begin{array}{c}\text { Error } \\
10^{-4}\end{array}$ & Order & $\begin{array}{c}N=40 \\
\frac{\text { Error }}{10^{-4}}\end{array}$ & Order & $\begin{array}{c}N=80 \\
\frac{\text { Error }}{10^{-4}}\end{array}$ & Order & $\begin{array}{c}N=160 \\
\frac{\text { Error }}{10^{-4}}\end{array}$ & Order \\
\hline$L_{2}$ & 127.7914 & 51.171 & 1.32 & 17.467 & 1.55 & 4.591 & 1.93 & 1.490 & 1.62 \\
$L_{\infty}$ & 181.7607 & 108.029 & 0.75 & 40.409 & 1.42 & 10.364 & 1.96 & 7.276 & 0.51
\end{tabular}

Table A.3. $L_{2}$ - and $L_{\infty}$-errors as functions of resolution and the spatial order of accuracy for the traveling-wave case with $c>0, Q=0$. In the interior, the polynomial order is $d_{P}=1$, and at the free boundary, a fractional basis function is used. Solutions are considered at dimensionless time $t=0.8$.

\section{A.4 Convergence tables}

To determine the spatial accuracy, the $L_{2}$ norm of the error has been used (and approximated by taking the mean values in each element)

$$
L_{2}=\sqrt{\frac{1}{H_{\text {wet }}} \int_{0}^{H_{\text {wet }}}\left(b_{\text {numerical }}-b_{\text {exact }}\right)^{2} \mathrm{~d} z},
$$

with $H_{\text {wet }}$ the inundated, open part of the domain; and also the $L_{\infty}$ norm, the maximum absolute difference between the numerical and the "exact" solution is considered.

Table A.1 shows that the spatial accuracy of the steady state solution in Fig. 4.8 reduces to order 1 for the $L_{2}$-error and order 0.5 for the $L_{\infty}$-error in the stationary case because of the steep gradient and the choked-flow condition, both at $z=H$. In Fig. 4.8, this discrepancy is observed at $z=H=3 \mathrm{~km}$, where the largest pointwise error occurs. Such a reduction of order is also observed in solutions with shocks or discontinuities. The exact and numerical traveling wave solution is shown in Fig. 4.9. Table A.2 shows that the spatial accuracy is approximately 1.5 to 1.64 for the traveling-wave case, as opposed to the expected, formal second-order accuracy, although error estimates vary between order one and two $[3]$.

The exact and numerical traveling-wave solution with a free boundary is provided in Fig. 4.10. The $L_{2}$-error has an order of 1.6, while the $L_{\infty}$-error behaves poorly and fluctuates heavily, see Table A.3. The actual time of splitting an element in relation to the measurement time makes the latter error a poor indicator of the order of convergence. 


\section{B Simulations}

In this Appendix, we introduce the numerical progams used to produce our results. Use of these programs is at one's own risk, and this section is added to provide general information and promote sharing ${ }^{1}$.

The c-programs, Matlab, and gnuplot files are all put together in a compressed tar file localsdg04.tar . Under linux or unix, after decompressing, the untarring creates the directory localsdg04 with all files in the unfolded directory named localsdg04. Use > make slcdq to compile and create the executable slcdq. The file Makefile can be used to alter the compilation settings to local circumstances. O.B. noticed that breaking a long formula caused compile errors on different machines and after compiler updates. These errors may have to be removed.

Matlab files, c-programs, gnuplot and input files are found in the directory $>/ \ldots /$ localsdg04 . eps-files have extension .eps, Matlab files extension .m , c-programs extension .c , and gnuplot files extension .gnu . The file cddat.m calculates most of the nondimensional parameters defined in the article and used in the figures. It can be used to check the values quoted. The eps-files used in the article are not present in the directory localsdg04, but cam be produced.

- The sketches in Fig. 2.1 and Fig. 3.1 are the files sketchlav0.eps and elemergsp.eps in directory $>/ \ldots$ /elasd .

- The functions displayed in Fig. 4.1 are found in stationsketb.eps in >/.../elasd, and made my the Matlab file statskcd3.m in $>/ \ldots$ /localsdg04. The addition of arrows and the placement of labels are done by hand.

- The functions displayed in Fig. 4.2 for constant $\alpha>0$ are statqd0ag0acb.eps and statzdag0acb.eps in directory $>/ \ldots$ /elasd, and made by statcd.m in $>/ \ldots /$ localsdg04. The functions displayed in Fig. 4.3 for constant $\alpha<0$ are statqd0al0acb.eps and statzdal0acb.eps in directory $>/ \ldots$ elasd , and made by statcd.m in $>/ \ldots /$ localsdg04 . By choosing the density in the rock $\rho_{r}=2800 \mathrm{~kg} / \mathrm{m}^{3}$ $(\alpha>0)$ or $\rho_{r}=2600 \mathrm{~kg} / \mathrm{m}^{3}(\alpha>0)$ in file statcd.m, we get the respective cases. Labels (a) and (b) are added in later by hand.

- The functions displayed in Fig. 4.4 are statzaz_qd0ac.eps an statzaz_zdac.eps in directory $>/ \ldots$. elasd , and made by statcdaz.m in $>/ \ldots /$ localsdg04 .

- The functions displayed in Fig. 4.5 are kamchatcd-ela0p95_3b.eps and kamchatcd-ela0p95_3pres.eps in directory $>\ldots$ /elasd, and made by running the files statcdzakamq.m and hydraulic03.m in $>\ldots$. localsdg04 consecutively.

- The sketch displayed in Fig. 4.6 is travsket.eps from directory $>/ \ldots$ /elasd, and is made by running the file travskcd3.m in $>/ \ldots$ /localsdg04 . Arrows are added in later by hand.

- The profiles displayed in Fig. 4.7 are traviii8b.eps, travi2b.eps, travii4b.eps, travii3b.eps, and traviii6b.eps from directory $>/ \ldots$ /elasd, and are made by running the files trav2cd.m for the choices $8,2,4,3,6$ of the variable choice $=8,2,4,3,6$ in $>/ \ldots /$ localsdg04 .

- The time dependent solution approaching the choked-flow steady state solution (the dashed line in Fig. 4.2b) is shown in Fig. 4.8 using file sdgstat40ci_altb.eps

In file ru.m most of the following Matlab commands have been typed in with the corresponding figure number indication. By taking away the comments the relevant command becomes active. It is made by running the c-program $>$ slcdq $<$ Icdci

The boundary conditions are set in the file init-Icdq.c under the function init_var_ci

Plotting is done with lcd1.m with the matlab command using the names of the output files, for example, r.lcdci2, b.Icdci2 specified in the input file Icdci:

$>>$ Icd1('r.lcdci2', 'b.Icdci2', 2,3,1,1,1080,1,1,0,1.2,0,2,0,6,0,2)

- The time dependent solution approaching the traveling-wave solution (the dashed line in Fig. 4.7c) with $c>0, Q<0$ with $b<b_{*}$ and $b_{0}<b<b_{1}$ is shown in Fig. 4.9 using file sdgtrav10t2d_altb.eps It is made by running the c-program $>$ slcdq $<$ lcdt2d

The boundary conditions are set in the file init-Icdq.c under the function init_var_t2d

1 This appendix is additional and not part of the formal publication "Magma Flow through Elastic-Walled Dikes" by O. Bokhove, A.W. Woods, and A. de Boer. 
Plotting is done with lcd1.m with the matlab command:

$>>$ Icd1('r.Icdt2d','b.Icdt2d', 3,3,1,0.1,1.0800e+06,1,1,0.8,2.2,0,0.06,0,6,0,2)

- The time dependent solution approaching the traveling-wave solution (the dashed line in Fig. 4.7a) with $c>0, Q=0$ is shown in Fig. 4.10 using file sdgtrav20t0p8tf0_altb.eps

It is made by running the c-program $>$ slcdq $<$ Icdtf0 with among others the choices: bctyperight $=$ Dirichlet, ic $=$ tff, tmax $=3$, nel $=20$

The boundary conditions are set in the file init-Icdq.c under the function init_var_tff

Plotting is done with lcd1.m with the matlab command:

$>>$ Icd1('r.Icdtf06','b.Icdtf06',14,3,1,1,1.0800e+03,1,1,0,1.,0,3,0,6,0,1)

- The variation on the above simulation with a choked-boundary condition once the traveling wave reaches the exit, is seen in Fig. 4.12 as file sdgtrav80t0p8tf0_chokt8_alteb.eps

It is made by running the c-program >slcdq < Icdtf0 (only working in program directory localsdg04130804.tar from 13-08-2004 momentarily) with among others the choices: bctyperight $=$ choked, ic $=$ tff, tmax $=3$, nel $=80$

The boundary conditions are set in the file init-Icdq.c under the function init_var_tff

Plotting is done with lcd1.m with the matlab command:

>>Icd1('r.Icdtf03','b.Icdtf03',14,3,1,1,1.0800e+03,1,1,0,1.,0,3,0,6,0,1)

The gnuplot program pl.gnu is used for one of the choices of the variable choi; this readily follows by spotting thee name of the data file used as input. That is, start gnuplot using the command gnuplot; define the variable nps (0 means no postscript output, 1 means postscript output), and choi, and then type the command load 'pl.gnu .

- The simulation in Fig. 4.13 in file kamtime80_t200h36lcdkacb.eps is made is made by running the c-program $>$ slcdq $<$ Icdke with the bctyperight $=$ Choked condition and choice ic $=$ ke

The boundary conditions are set in the file init-Icdq.c under the function init_var_ke

Plotting is done with lcd1.m with the matlab commands: >>statcdzakamq.m

for the steady state limiting solution and setting nfigg $=1$ and $\mathrm{Nq}=1$ and then

$>>\operatorname{lcd} 1$ ('r.lcdke','b.lcdke', 20,3,1,1,1.0800e+03,1,1,0,2,0,3,0,6,0,1)

- The simulations in Fig. 4.14 in files lavasinkt1p8r90.eps and avasinkt5r90b.eps are made by running the c-program $>$ slcdq $<$ Icdsh with the bctyperight $=$ Choked condition and choice ic $=$ sh

The boundary conditions are set in the file init-lcdq.c under the function init_var_sh

Plotting is done with Icdq.m >> Icd1('r.Icdsh3', 'b.Icdsh3',44,3,1,1,2.160,1,1,0,1.5,0,3,0,6,0,1)

- The sketch in Fig. 4.15 is file dome.eps.

- The simulation in Fig. 4.16 uses the file stlcdsou.eps

It is made by running $>$ slcdq $<$ Icdsou

Plotting is done by using $>>\operatorname{Icd} 1$ ('r.Icdsou', 'b.Icdsou', $20,10,0.0833,1,1200,1,1,-1,1,0,16,0,6,1,1)$

The gnuplot program pl.gnu is used for one of the choices of the variable choi; this readily follows by spotting thee name of the data file used as input. Take care the right name of the data file is set. 